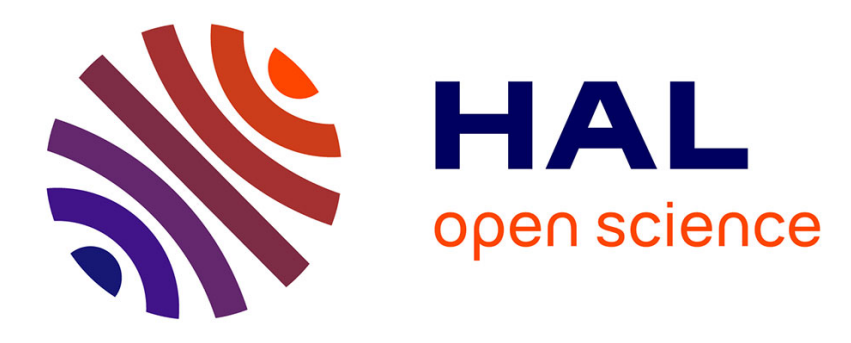

\title{
A numerical study of attraction/repulsion collective behavior models: 3D particle analyses and 1D kinetic simulations
}

Francesco Vecil, Pauline Lafitte, Jesus Rosado Linares

\section{- To cite this version:}

Francesco Vecil, Pauline Lafitte, Jesus Rosado Linares. A numerical study of attraction/repulsion collective behavior models: 3D particle analyses and 1D kinetic simulations. Physica D: Nonlinear Phenomena, 2013, 10.1016/j.physd.2012.12.010. 10.1016/j.physd.2012.12.010 . hal-00726486

\section{HAL Id: hal-00726486 https://hal.science/hal-00726486}

Submitted on 30 Aug 2012

HAL is a multi-disciplinary open access archive for the deposit and dissemination of scientific research documents, whether they are published or not. The documents may come from teaching and research institutions in France or abroad, or from public or private research centers.
L'archive ouverte pluridisciplinaire HAL, est destinée au dépôt et à la diffusion de documents scientifiques de niveau recherche, publiés ou non, émanant des établissements d'enseignement et de recherche français ou étrangers, des laboratoires publics ou privés. 


\title{
Numerical analysis on attraction/repulsion collective behavior models: particle and kinetic approaches
}

\author{
Francesco Vecil, \\ francesco.vecil@gmail.com \\ Universitat de València \\ Pauline Lafitte, \\ pauline.lafitte@ecp.fr \\ INRIA Lille Nord Europe, EPI SIMPAF \& École Centrale Paris, Lab. MAS, \\ Jesús Rosado Linares, \\ jrosado@math.ucla.edu \\ UCLA Mathematics Department
}

\begin{abstract}
We study at particle and kinetic level a collective behavior model based on three phenomena: self-propulsion, friction (Rayleigh effect) and an attractive/repulsive (Morse) potential rescaled so that the total mass of the system remains constant independently of the number of particles $N$. In the first part of the paper, we introduce the particle model: the agents are numbered and described by their position and velocity. We identify five parameters that govern the possible asymptotic states for this system (clumps, spheres, dispersion, mills, rigid-body rotation, flocks) and perform a numerical analysis on the $3 \mathrm{D}$ setting. Then, in the second part of the paper, we describe the kinetic system derived as the limit from the particle model as $N$ tends to infinity; we propose, in 1D, a numerical scheme for the simulations, and perform a numerical analysis devoted to trying to recover asymptotically patterns similar to those emerging for the equivalent particle systems, when particles originally evolved on a circle.
\end{abstract}

Keywords : collective behavior, self-organization, swarming, attractive/repulsive potential MSC 92B05, 70F99, 65P40, 35L50

\section{Introduction}

Collective behaviors arise when a set of individuals organize into macroscopically observable patterns without the active role of a leader, rather by self-organization. As examples we can 
give the diffusion of languages in primitive societies [T], the averaging of prices in a stock exchange, or in biology the movement of bird flocks [2, 3], fish schools [4, 5, 6], insect swarms [7], sheep herds and even some micro-organisms [8]. Application of collective behavior theory includes various fields: for instance, in engineering, it can be used to coordinate robots or autonomous vehicles for unmanned operations, see [9, 10, 3, 11, 12, 1:3] and included references. Another example is their use in sociology to predict criminal behavior, see [14] for instance.

Depending on the number of agents taken into account, microscopic [15, 16, 17, 18] or macroscopic [15, 20, 21, 22] models can be used: the first ones go by the name of discrete, or particle, or individual-based models, and in them the agents are numbered and identified by their position and velocity, plus any other feature which might be considered useful for the proposed goal, for instance the size of cells or the age of human beings; moreover, the models must include the dynamics, the rules describing the behavior of the agents, like the tendency animals have to group together, nonetheless repelling each other when they are too close. When the number of agents is very large, for example when studying the migration of fish schools involving millions of individuals, numerical simulations become unaffordable, therefore macroscopic models have to be used, divided essentially into two categories: kinetic and hydrodynamic. The macroscopic models might be written directly following phenomenological rules [2, 20, 19, 2:3]. Otherwise, kinetic models can be derived from individual-based models, as in [24, 25, 26, 27, 28] where a formal derivation from the microscopic model is obtained, and qualitative properties are studied. In [2.9], the authors are able to reduce the dimensionality of the mesoscopic model by constraining the velocities on a sphere $\mathbb{S}^{d}$, in the spirit of the Vicsek model [30]. Hydrodynamic models, also derived from individual-based models, are used to reduce even more the dimensionality of the system; see [3], 27, 26, 32, 33. 34, 35].

The goal of this work is to investigate numerically a model reflecting self-propulsion, friction and the attraction/repulsion phenomena. The particles we are considering can model a variety of physical and biological situations, such as fish, polymers, and so on. One can find a review in [34]. Let $t \geq 0$ be the time variable and $d \in\{1,2,3\}$ the dimension of spaces for position and velocity. Assuming that there are $N$ particles of masses $\left\{m_{i}\right\}_{i=1, \ldots, N}$, Newton's second law reads, for all $i \in\{1, \ldots, N\}$,

$$
\begin{aligned}
& \frac{d}{d t} \boldsymbol{x}^{(i)}=\boldsymbol{v}^{(i)} \\
& m_{i} \frac{d}{d t} \boldsymbol{v}^{(i)}=\underbrace{m_{i} \alpha \boldsymbol{v}^{(i)}}_{\text {self-propulsion }}-\underbrace{m_{i} \beta\left|\boldsymbol{v}^{(i)}\right|_{\mathbb{R}^{d}}^{2} \boldsymbol{v}^{(i)}}_{\text {friction (Rayleigh) }}-\underbrace{m_{i} \sum_{j \neq i} m_{j} \nabla_{x} U\left(\boldsymbol{x}^{(i)}-\boldsymbol{x}^{(j)}\right),}_{\text {attractive/repulsive (Morse) potential }}
\end{aligned}
$$

where the potential $U$ is defined as:

$$
U(\boldsymbol{x})=-C_{a} W\left(\boldsymbol{x} / \ell_{a}\right)+C_{r} W\left(\boldsymbol{x} / \ell_{r}\right)
$$

with $W: \boldsymbol{z} \mapsto \exp \left(-|\boldsymbol{z}|_{\mathbb{R}^{d}}^{p}\right), p \geq 1$. As in the gravitational or Coulombian models of interaction, we adopt a quadratic dependency on the mass for the attraction/repulsion force. 
From now on, we assume that the particles are indiscernible $\left(m_{i}=M / N\right)$, so that the total mass $M$ is the only mass parameter of the system; furthermore, we shall normalize the system so that $M=1$ in our simulations. The self-propulsion and friction constants per mass unit $\alpha$ and $\beta$, the characteristic lengths of attraction and repulsion $\ell_{a}$ and $\ell_{r}$ and the amplitude constants $C_{a}$ and $C_{r}$ are given data.

We are interested in comparing the numerical results obtained in this microscopic description with simulations derived at a mesoscopic level. In other words, based on a kinetic equation, we will describe the evolution of the particles from a statistical point of view as a function of the time $t \geq 0$, the position $\boldsymbol{x} \in \mathbb{R}^{d}$ and the velocity $\boldsymbol{v} \in \mathbb{R}^{d}$. Let $f(t, \boldsymbol{x}, \boldsymbol{v})$ be the distribution function of the particles, that is, at time $t$, in a volume $\Omega \in \mathbb{R}^{d} \times \mathbb{R}^{d}$, there are $\int_{\Omega} f(t, \boldsymbol{x}, \boldsymbol{v}) \mathrm{d} \boldsymbol{x} \mathrm{d} \boldsymbol{v}$ particles. Let $\rho(t, \boldsymbol{x})=\int_{\mathbb{R}^{d}} f(t, \boldsymbol{x}, \boldsymbol{v}) \mathrm{d} \boldsymbol{v}$ be the macroscopic concentration of particles at time $t$ and position $\boldsymbol{x}$. The kinetic equation, obtained from (四) as a mean-field limit [36] , reads

$$
\partial_{t} f+\underbrace{\boldsymbol{v} \cdot \boldsymbol{\nabla}_{\boldsymbol{x}} f}_{\text {free motion }}+\underbrace{\nabla_{\boldsymbol{v}} \cdot\left[\left(\alpha-\beta|\boldsymbol{v}|_{\mathbb{R}^{d}}^{2}\right) \boldsymbol{v} f\right]}_{\text {self-propulsion and friction }}-\underbrace{\nabla_{\boldsymbol{v}} \cdot\left[\left(\boldsymbol{\nabla}_{\boldsymbol{x}} U * \rho\right) f\right]}_{\text {Morse potential }}=0,
$$

with $\int_{\mathbb{R}^{d}} \rho \mathrm{d} x=M$. We assume that $f(0, \cdot, \cdot) / M \in \mathcal{P}^{1}\left(\mathbb{R}^{d} \times \mathbb{R}^{d}\right)$, that is, a probability measure with finite first moment, and we moreover assume that it has a compact support. It was proved by Cañizo, Carrillo and Rosado [32] that, at least in the case $p=2$, the solution $f / M$ lies in $\mathcal{C}\left([0,+\infty), \mathcal{P}^{1}\left(\mathbb{R}^{d} \times \mathbb{R}^{d}\right)\right)$. The model was originally studied for the Morse potential $(p=1)$ in [17] for particles and later on, in [36] at a kinetic level.

The effect of the self-propulsion/friction part will be to fix the modulus of the velocities to $\sqrt{\alpha / \beta}$, and that of the Morse potential to make the particles repel each other when they are too close, and attract when they are far, roughly fixing relative distances corresponding to its minimum and thus favoring the formation of crystalline structures. According to [17], the dimensionless quantities of interest are $C=C_{r} / C_{a}$ and $\ell=\ell_{r} / \ell_{a}$, that lead to the formation of different patterns. We study how the hierarchy of three other quantities, namely the characteristic times associated to the transport term $t_{k i n}=\ell_{a} \sqrt{\beta / \alpha}$, to the selfpropulsion/friction term $t_{f / p}=1 / \alpha$ and to the Morse term $t_{a / r}=\ell_{a} /\left(C_{a} M\right) \sqrt{\alpha / \beta}$, is also relevant in order to predict the behavior. Of course, in order to present a readable approach of this multiparameter model, we focused our attention on a restricted number of cases.

This paper is organized as follows: in Section 27 our reference model at particle level is introduced, adimensionalized and a numerical study on its properties is performed in the 3D setting paying attention to the fact that the dimension is higher that what can be found in the literature and the potential is rescaled; in Section 3 we detail the kinetic model corresponding to the discrete one as the mean-field limit $N \rightarrow \infty$, we propose a numerical scheme to solve it and perform numerical tests in the 1D periodic case; finally, in Section $⿴$ we present our conclusions and plans for the future. 


\begin{tabular}{|c|c|c|c|c|c|c|c|c|c|c|c|c|c|c|}
\hline Case nr. & $\alpha$ & $\beta$ & $C_{r}$ & $\ell_{r}$ & $C_{a}$ & $\ell_{a}$ & $t_{k i n}$ & $t_{f / p}$ & $t_{a / r}$ & $C$ & $\ell$ & $C \ell^{3}$ & $C \ell$ & Figs \\
\hline MKR-I & 1 & .5 & 60 & .5 & 100 & 1 & .70 & 1 & .14 & .6 & .5 & 7 & $T$ & $\begin{array}{c}18(b) \\
19(a)\end{array}$ \\
\hline MKR-II & 1 & .5 & 50 & .5 & 100 & 1 & .70 & 1 & .14 & .5 & .5 & 7 & 7 & $\begin{array}{c}\text { 而 } \\
18(\mathrm{c}) \\
19(\mathrm{~b})\end{array}$ \\
\hline MKR-III & 1 & .5 & 40 & .6 & 100 & 1 & .70 & 1 & .14 & .4 & .6 & 7 & 7 & $5(b) \ldots$ \\
\hline MKR-IV & 1 & .5 & 50 & 1.2 & 100 & 1 & .70 & 1 & .14 & .5 & 1.2 & 1 & / & (c) $\ldots$ \\
\hline MKR-VI-1 & .1 & 5.0 & 350 & .8 & 100 & 1.2 & 8.48 & 10 & .0016 & 3.5 & .66 & 1.006 & 2.31 & $\begin{array}{c}\mathbf{0} \\
\mathbf{\square} \\
8(\mathrm{a}) \\
8(\mathrm{~b}) \\
18(\mathrm{~d}) \\
19(\mathrm{c})\end{array}$ \\
\hline MKR-VII & 1 & .5 & 60 & .5 & 50 & 1 & .71 & 1 & .03 & 1.2 & .5 & .15 & .6 & प्य \\
\hline MRK-I-2 & .07 & .05 & 50 & 2 & 20 & 100 & 84.52 & 14.29 & 1.18 & .5 & .02 & / & / & ए.3 \\
\hline MRK-VI & 1 & 5.0 & 350 & .8 & 100 & 1.2 & 2.68 & 1 & .0053 & 3.5 & .66 & 1.006 & 2.31 & $\nabla(\mathrm{c})$ \\
\hline MRK-VII-1 & .2 & .1 & 500 & 2 & 200 & 100 & 7.71 & 5 & .70 & 2.5 & .02 & $2.0 \mathrm{e}-5$ & .05 & पा \\
\hline MRK-VII-2 & .07 & .05 & 50 & 2 & 20 & 100 & 84.52 & 14.29 & 5.92 & 2.5 & .02 & $2.0 \mathrm{e}-5$ & .05 & 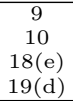 \\
\hline MRK-VII-3 & $3.5-5.5$ & 5.0 & 150 & .8 & 100 & 1.2 & $1.43-1.14$ & $.28-.18$ & $(1.0-1.2) \mathrm{e}-2$ & 1.5 & .66 & .43 & .99 & ए2 \\
\hline KMR-VII & .07 & .05 & 2.5 & .02 & 1 & 1 & .84 & 14.29 & 1.18 & 2.5 & .02 & $2.0 \mathrm{e}-5$ & .05 & $\begin{array}{l}15(\mathrm{a}) \\
18(\mathrm{f}) \\
19(\mathrm{e})\end{array}$ \\
\hline KRM-VII & 1.4 & .05 & 2.5 & .02 & 1 & 1 & .19 & .71 & 5.29 & 2.5 & .02 & $2.0 \mathrm{e}-5$ & .05 & $\begin{array}{l}15(\mathrm{~b}) \\
18(\mathrm{~g}) \\
19(\mathrm{f})\end{array}$ \\
\hline RMK-VII & 20 & .5 & 60 & .5 & 50 & 1 & .16 & .05 & .13 & 1.2 & .5 & .15 & .6 & $16(\mathrm{a})$ \\
\hline RKM-VII & 1.4 & .05 & 50 & 2 & 20 & 100 & 18.89 & .71 & 26.45 & 2.5 & .02 & $2.0 \mathrm{e}-5$ & .05 & $16(\mathrm{~b})$ \\
\hline
\end{tabular}

Table 1: List of parameters. The names are given under the following philosophy: the three letters rank the typical kinetic $(\mathrm{K})$, self-propulsion/friction (R for Rayleigh) and attraction/repulsion ( $\mathrm{M}$ for Morse) times; the Roman numerals describe the region as in diagram Figure W; if several cases have the same time and region rankings, they are numbered with Arabic numerals.

\section{The particle model}

In this section we describe in detail the particle model and perform a numerical analysis to have an intuition on the behavior in the limit $N \rightarrow \infty$ for different regimes; this helps us understand whether the continuum model is meaningful or not. We set ourselves in the 3D space $d=3$.

We list in Table 2 all the parameters used for the simulations, the meaning of the characteristic times being explained in detail in the following paragraph.

\subsection{Newton laws for the individual motion}

The model in which we are interested for the scope of this work is given by the following Newton laws:

$$
\begin{aligned}
\frac{d}{d t} \boldsymbol{x}^{(i)} & =\boldsymbol{v}^{(i)} \\
\frac{d}{d t} \boldsymbol{v}^{(i)} & =\alpha \boldsymbol{v}^{(i)}-\beta\left|\boldsymbol{v}^{(i)}\right|^{2} \boldsymbol{v}^{(i)}-\frac{1}{N} \sum_{j \neq i} \nabla U\left(\boldsymbol{x}^{(i)}-\boldsymbol{x}^{(j)}\right) .
\end{aligned}
$$


as described in (四). Here

$$
[0,+\infty) \longrightarrow \mathbb{R}^{3} \times \mathbb{R}^{3}, \quad t \mapsto\left(\boldsymbol{x}^{(i)}(t), \boldsymbol{v}^{(i)}(t)\right)
$$

represent the position and the velocity of the individual number $i$ at time $t$. The term $\alpha \boldsymbol{v}^{(i)}$ in the equations of motion represents the self-propulsion: if it was not balanced by other terms, it would make the individuals accelerate along the velocity they have at time $t$. The contribution given by the term $-\beta\left|\boldsymbol{v}^{(i)}\right|^{2} \boldsymbol{v}^{(i)}$ is called friction or Rayleigh dissipation and has been firstly introduced in [37]: its sign is the opposite of the self-propulsion's and balances its effects; together, these two terms asymptotically fix the modulus of the velocity of the individual number $i$ to $\sqrt{\alpha / \beta}$ [2, 5. 38, 3.9, 40], as can be seen in the computations of the characteristics that is performed further on (see $\mathbb{A}$ ). The potential $U$ [41, 42] is a typical pairwise potential which states that two individuals tend to repel (resp. attract) each other when they are close to the typical repulsion length $\ell_{r}$ (resp. attraction length $\ell_{a}$ ). In the middle region between repulsion and attraction, there might be a zone called of alignment, where individuals tend to mimic the behavior of their neighbors; although this model does not have a specific term modeling this effect, in [34] it is shown that, in 2D, an alignment occurs nevertheless for an appropriate choice of parameters. The parameters $C_{r}$ and $C_{a}$ describe the strength of repulsion and attraction respectively. A typical choice for this attractive/repulsive potential is [15, 16, 34]

$$
U(\boldsymbol{x})=-C_{a} \exp \left(-\frac{|\boldsymbol{x}|^{p}}{\ell_{a}^{p}}\right)+C_{r} \exp \left(-\frac{|\boldsymbol{x}|^{p}}{\ell_{r}^{p}}\right)
$$

with $p \in\{1,2\}$. In the following, we want to identify the parameters that will influence the asymptotic behavior of the solution. Following the same procedure as in [34], we introduce the rescalings

$$
\tilde{\boldsymbol{x}}=x_{*} \boldsymbol{x}, \quad \tilde{t}=t_{*} t, \quad \tilde{\boldsymbol{v}}=v_{*} \boldsymbol{v}
$$

where magnitudes with tilde are meant as being dimensional. Recalling that the friction/selfpropulsion effect is to force the asymptotic velocity to be of modulus $\sqrt{\alpha / \beta}$, we choose $v_{*}=\sqrt{\alpha / \beta}$, so that, in the dimensionless setting, the limiting velocity will be of modulus 1 . Moreover, we choose $x_{*}=\ell_{a}$ so that the ratio $\ell$ will play a key role. Making the respective characteristic times $t_{k i n}, t_{f / p}$ and $t_{a / r}$ appear, the dimensionless system reads

$$
\frac{d \boldsymbol{x}^{(i)}}{d t}=\underbrace{\frac{t_{*}}{t_{k i n}} \boldsymbol{v}^{(i)}}_{\text {free motion }}, \quad \frac{d \boldsymbol{v}^{(i)}}{d t}=\underbrace{\frac{t_{*}}{t_{f / p}}\left(1-\left|\boldsymbol{v}^{(i)}\right|^{2}\right) \boldsymbol{v}^{(i)}}_{\text {self-propulsion and friction }}-\underbrace{\frac{t_{*}}{t_{a / r}} \frac{1}{N} \sum_{j \neq i} \boldsymbol{\nabla}_{\boldsymbol{x}}[-W(\boldsymbol{x})+C W(\boldsymbol{x} / \ell)]}_{\text {Morse potential }}
$$

where

$$
t_{k i n}=\ell_{a} \sqrt{\frac{\beta}{\alpha}}, \quad t_{f / p}=\frac{1}{\alpha}, \quad t_{a / r}=\frac{\ell_{a}}{C_{a} M} \sqrt{\frac{\alpha}{\beta}} .
$$

We have reduced the initial seven parameters of the model $\left(M, \ell_{a}, \ell_{r}, C_{a}, C_{r}, \alpha, \beta\right)$ and the observation length, time and velocity to three characteristic time ratios $t_{*} / t_{k i n}, t_{*} / t_{f / p}$ and $t_{*} / t_{a / r}$ and two characteristic spatial ratios $C=C_{r} / C_{a}$ and $\ell=\ell_{r} / \ell_{a}$. 
Remark 2.1. If the characteristic-time ratios are very large or very small, we might be able to predict the asymptotic behavior of the system according to the dominant term.

The biologically relevant situation is $\{C>1\} \cap\{\ell<1\}$, which means that the typical repulsion length is shorter than the typical attraction length and that the force trying to keep the individuals together is stronger that the force trying to make them reject each other at short distances, thus avoiding dispersion. This situation, the typical behavior of animal species, goes by the name of short-range repulsion and long-range attraction. From [34] we know that a criterion called H-stability is necessary and sufficient for the existence of thermodynamics; in other words, a criterion under which the limit $N \rightarrow \infty$ is meaningful, because it guarantees that the density of particles remains bounded. In the catastrophic case, the radius of the cloud of particles, defined as

$$
\mathcal{R}(t ; N)=\sup _{i=1, \ldots, N}\left|\boldsymbol{x}^{(i)}(t)-\boldsymbol{x}_{C M}(t)\right|_{\mathbb{R}^{d}},
$$

$\boldsymbol{x}_{C M}$ being the position of the center of mass, remains constant, thus yielding a blow up of the density. As the pairwise potential is the same as in [34] or [I7], the H-stability diagram will be the same up to taking into account that the setting is now $\mathbb{R}^{3}$ instead of $\mathbb{R}^{2}$. From [43] we know that the regime is catastrophic if $\int_{\mathbb{R}^{d}} U(x) \mathrm{d} x<0$, which is equivalent, in our

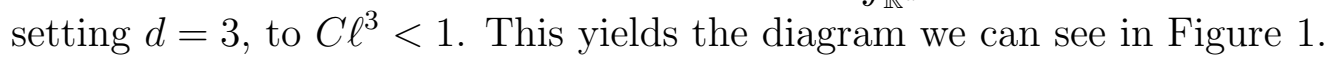

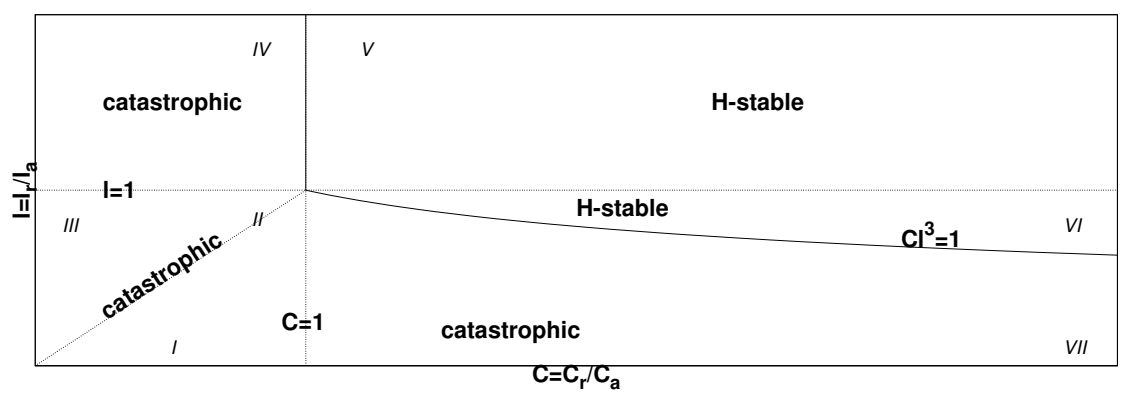

Figure 1: H-stability diagram for the Morse potential (『)).

Remark 2.2. We stress that in [34, 17] a non-rescaled pairwise potential is used, i.e. there is no factor $\frac{1}{N}$, which leads to quantitatively different behaviors.

\subsection{Patterns in $\mathbb{R}^{3}$ : Morse point of view}

In order to perform the individual-based model simulations, we use a third-order RungeKutta time discretization, the solver being uniformly randomly initialized inside a parallelepiped. As particle solvers do not require any boundaries, the particles are let free to 
move on an unbounded domain. In the following, we analyze how the regions in the diagram of Figure W, which represent properties of the Morse potential, affect the transient and asymptotic states of the system.

Notations. We shall use the following notation for the test cases: the three letters $\mathrm{K}$, $\mathrm{R}, \mathrm{M}$, in different orders, then a Roman numeral from I to VII; and, sometimes, an Arabic numeral. The three letters refer to the dominance in the typical times: $\mathrm{K}=$ kinetic, $\mathrm{R}=$ self-propulsion/friction (from Rayleigh), $\mathrm{M}=$ attraction/repulsion (from Morse). The Ro-

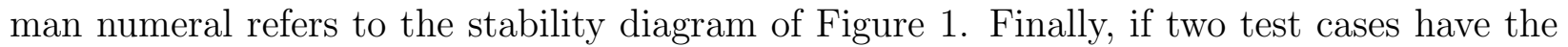
same typical-time hierarchy and belong to the same H-stability region, they are numbered from 1 onward. For instance, RMK-VI-2 means that $t_{f / p} \leq t_{a / r} \leq t_{k i n}$, that the parameters belong to region $\mathrm{VI}$ of the $\mathrm{H}$-stability diagram and that there are at least two benchmarks with these characteristics.

\subsubsection{Clumps}

In the region I of the diagram of Figure $\square$ we are in a regime where particles self-organize into small groups, which rotate around the center of mass. The behavior is similar to that of the 2D case observed in [17]; nevertheless, as we are in higher dimension, some more complex trajectories are allowed and the rotation of particles has a complex shape, as it is sketched in Figure $\nabla$ for two particles.

In Figure 3 we see that, as was the case in $2 \mathrm{D}$ in the catastrophic regime, the radius remains essentially constant.
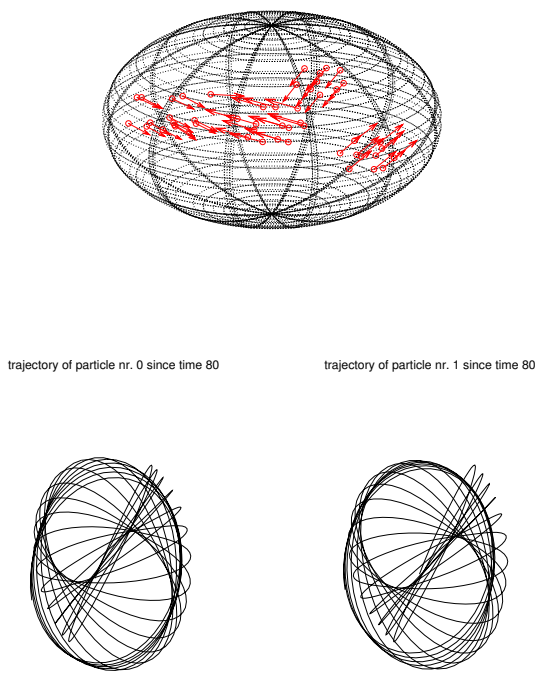

Figure 2: Clumps in 3D. Case MKR-I in Table 2 . 


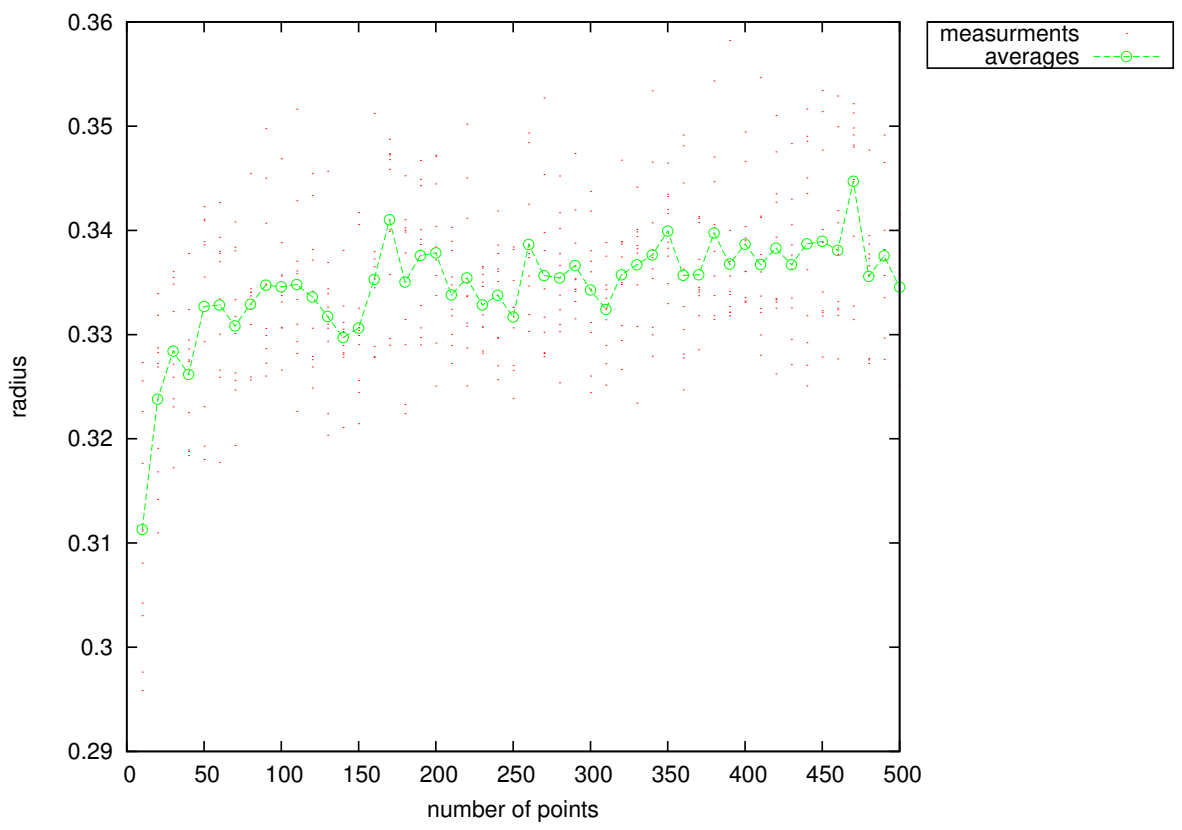

Figure 3: Clumps in 3D. Parameters are those of case MKR-I in Table 2. 


\subsubsection{Rings}

In the regime corresponding to region II of

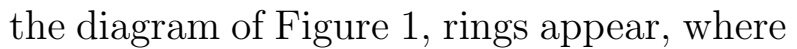
in this case "ring" means that the particles have the same distance to the center of mass, therefore they are rather "spheres", as can be seen in Figure 耳. As well as for the clumps, we observe no dependency of the radius on the number of particles, as sketched in the bottom part of Figure 5(a).

Rings are also obtained in regions III and IV of the diagram of Figure $\square$ with the same qualitative behavior as in region II. The evolution of the radius of the cloud of particles is drawn in Figure 5 .

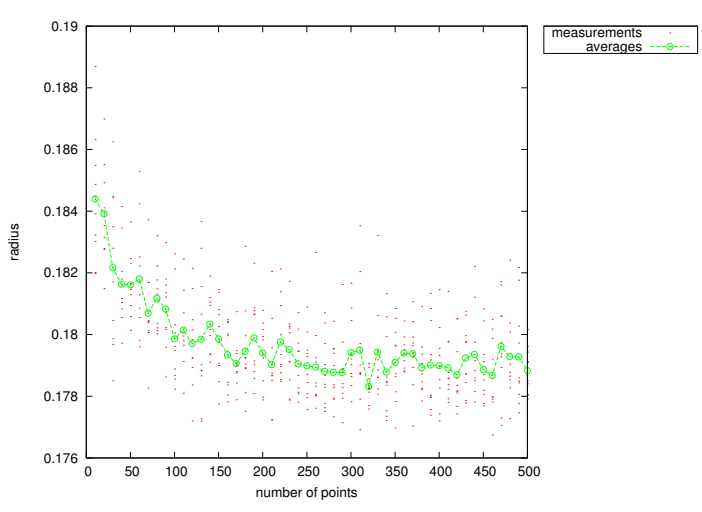

(a) radius of the $3 \mathrm{D}$ rings (region $\mathrm{II}$ )

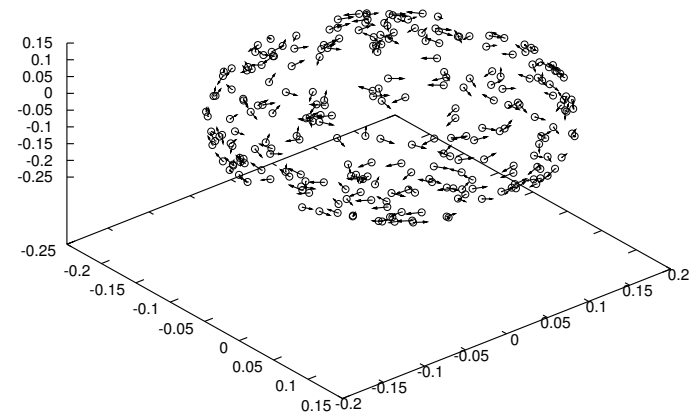

Figure 4: Rings in 3D. Case MKR-II in Table 2

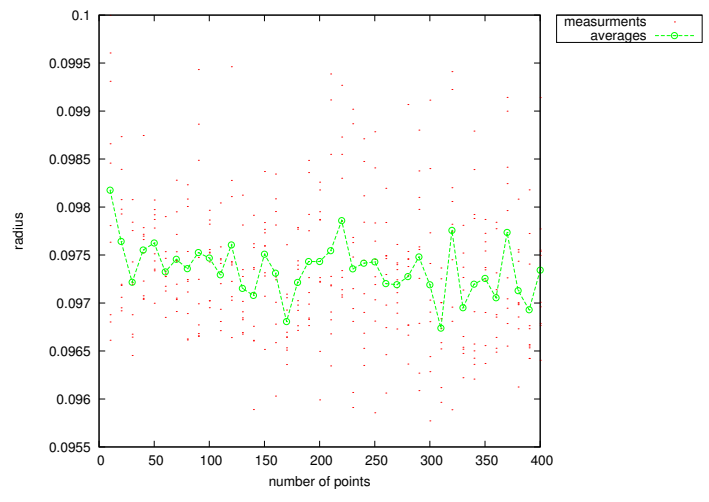

(b) radius of the rings (region III)

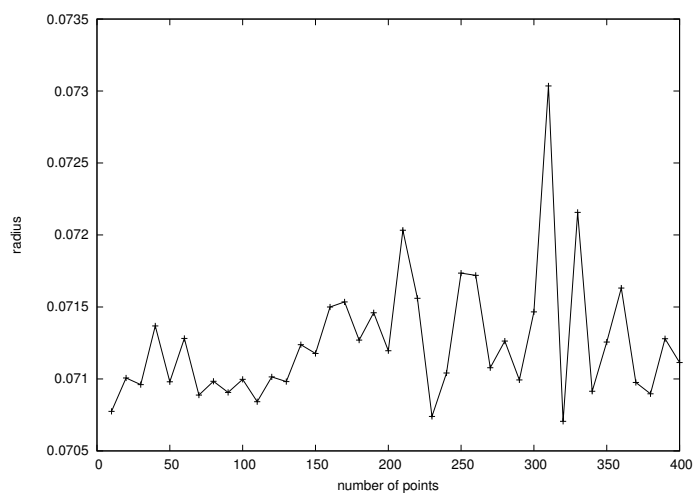

(c) radius of the rings (region IV)

Figure 5: Rings in 3D. For (a), parameters are those of case MKR-II in Table Q2. For (b), parameters are those of case MKR-III in Table 2 . For (c), parameters are those of case MKR-IV in Table 2 . 


\subsubsection{Gaseous behavior}

Region V, as observed in [17], corresponds to a gaseous region: the agents repel each other at any distance and will, therefore, try to occupy the whole volume.

\subsubsection{Lattice state}

By lattice state we mean a configuration in which the reciprocal distances are kept fixed; the clearest example is the coherent flock, in which all the particles travel with the same velocity $\overline{\boldsymbol{v}}$, a behavior which arises also in alignment models [3]. The coherent flock, with $|\overline{\boldsymbol{v}}|_{\mathbb{R}^{d}}^{2}=\alpha / \beta$, satisfies

$$
0=\frac{d \boldsymbol{v}^{(i)}}{d t}=\alpha \boldsymbol{v}^{(i)}-\beta\left|\boldsymbol{v}^{(i)}\right|^{2} \boldsymbol{v}^{(i)}-\frac{1}{N} \sum_{j \neq i} \boldsymbol{\nabla} U\left(\boldsymbol{x}^{(i)}-\boldsymbol{x}^{(j)}\right)=-\frac{1}{N} \sum_{j \neq i} \boldsymbol{\nabla} U\left(\boldsymbol{x}^{(i)}-\boldsymbol{x}^{(j)}\right),
$$

therefore, as remarked in [34], it is a traveling-wave solution for the model and a solution of the Euler-Lagrange equation

$$
\sum_{j \neq i} \nabla U\left(\boldsymbol{x}^{(i)}-\boldsymbol{x}^{(j)}\right)=0
$$

Region VI is likely to produce dispersed states, apart from small values of $\sqrt{\alpha / \beta}$, for which crystalline structures may appear, because the preferred reciprocal distance for the particles is $r=\frac{\log \left(\frac{C}{\ell}\right)}{\frac{1}{\ell_{r}}-\frac{1}{\ell_{a}}}$, zero of the Morse force field. The most probable pattern is a coherent flock, but unlike the 2D setting, where the particles adopt a uniform velocity quite rapidly, in the $3 \mathrm{D}$ setting particles maintain a rotational movement around the center of mass, thus mixing the coherent flock with a rigid-body rotation, i.e. the configuration in which particles keep fixed reciprocal distances while turning around the center of mass with constant angular velocity. The situation is sketched in Figure 6 . From that picture we also observe that this rotational movement is asymptotically fading: the upper and lower part of Figure [ depict the same time length, and while from time 1000 to time 1500 there is more that one complete twist around the center of mass, this is no more the case from time 4500 to time 5000 .

As the number of particles increases, the flock patterns become more likely. Anyway, the common point among all these formations is that the particles oscillate, in different ways, around the positions of a crystalline structure.

In Figure $\mathbf{7}$ (a) we can observe that the H-stability reflects in the increase of the volume as the number of particles grows, which seems to be consistent with the ratio seen in the $2 \mathrm{D}$ case; from the graphic, the increase in the number of agents seems to favor dispersed states: we can observe that for $\alpha=0.1$, from $N=300$ we obtain mainly dispersed states, while for $\alpha=0.01$ we still have swarming states. In Figure $\square$ (b) we plot the threshold between swarming states and dispersed states in this regime, where we see $\alpha_{\text {escape }}$ decreases with the number of particles because of the weakened effect of the rescaled potential, contrary to what is observed in [34]. 

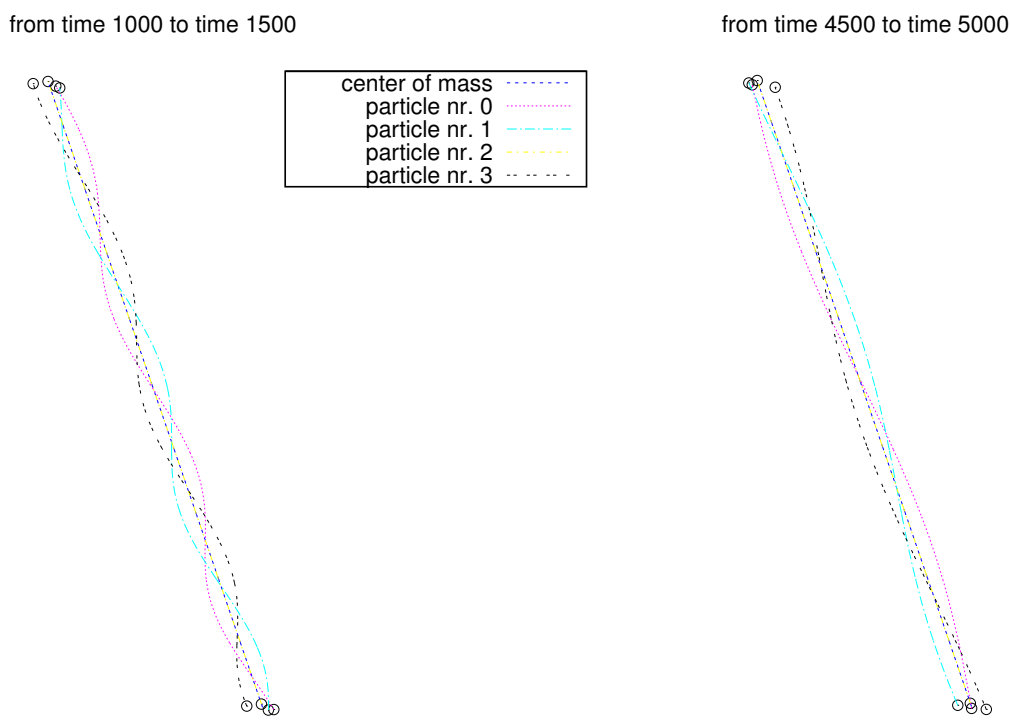

Figure 6: Spurious coherent flock in 3D. Particles achieve a uniform velocity only asymptotically, while during the transient state they couple the flock with a rigid-body rotation. Parameters are those of case MKR-VI-1 in Table 2 .

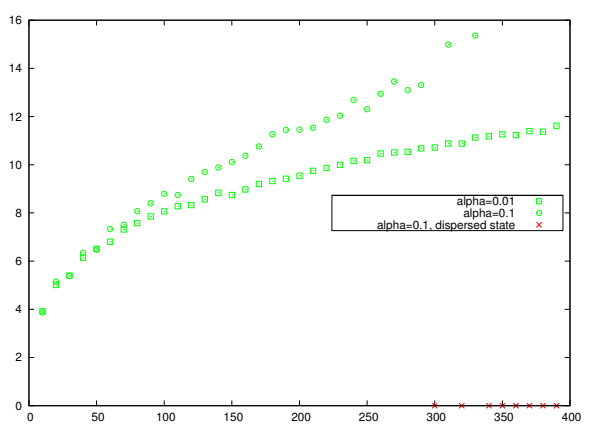

(a) radius

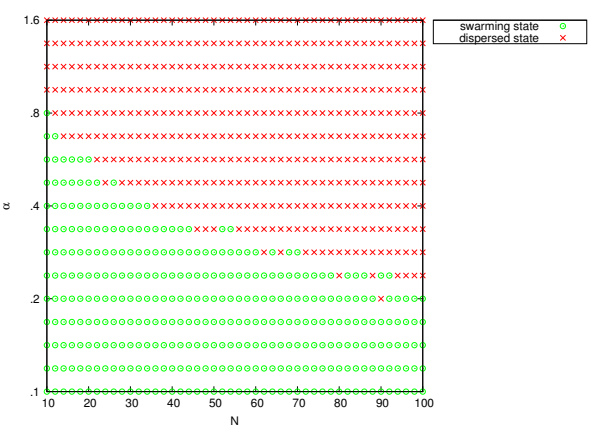

(b) $\alpha_{\text {escape }}$

Figure 7: Flock in region VI. Parameters are those of case MKR-VI-1 in Table घ (a) Evolution of the radius. (b) The threshold between swarming states and dispersed states. 


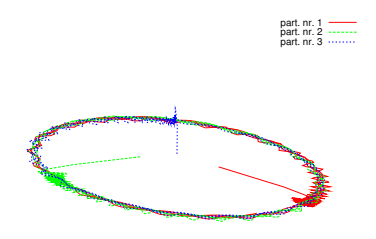

(a) mill

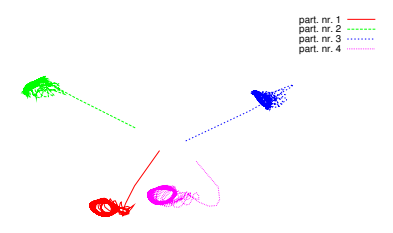

(b) multiple mills

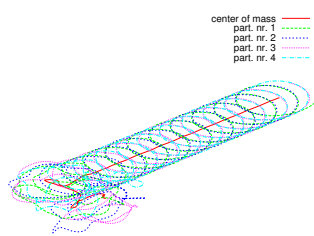

(c) helix

Figure 8: Patterns for low particle density in region VI. (a) and (b): parameters are those of case MKR-VI-1 in Table 2. (c): parameters are those of case MRK-VI in Table 2.

Remark 2.3. Even if the spirit of the present work is to study what happens in the limit $N \rightarrow \infty$, we remark how, for a small number of particles, we might observe some bizarre patterns like a 1-mill pattern (Figure $\mathbf{8}(a)$ ), four independent 1-mills (Figure $\mathbf{8}(b)$ ), or a helix evolution (Figure $\mathbf{\nabla}(c))$ about the trajectory of the center of mass.

\subsubsection{Region VII}

This region of the parameter space is the richest one in terms of patterns. As well as in region VI, coherent flock is a likely pattern, and as well as in region VI, it appears mixed with a rigid-body rotation.

By rotational state we mean a configuration in which particles move around the center of mass. Mills and rigid-body rotations are examples of this: in the first case all the particles travel with the same modulus of the velocity around an empty core, in the second one the particles keep fixed distances (as already said in Section 2.2.4). These two configurations may appear as different stages of the same simulation: in Figure $\mathbf{9}$ we see an example of this. After some initial oscillations, particles mill about a donut-shaped space, then they divide into smaller groups and behave more as a rigid body.

Instability of mills. - Numerical experiments suggest that mills are only a meta-stable state for the system: as a consequence, mills are just a transient state which may eventually be broken and degenerate into a coherent flock. In order to decide whether the observed pattern is a mill-state or a coherent flock, we use the measures in [34], called polarity and normalized angular momentum:

$$
P=\frac{\left|\sum_{i=1}^{N} \boldsymbol{v}^{(i)}\right|}{\sum_{i=1}^{N}\left|\boldsymbol{v}^{(i)}\right|}, \quad M=\frac{\left|\sum_{i=1}^{N} \boldsymbol{r}^{(i)} \wedge \boldsymbol{v}^{(i)}\right|}{\sum_{i=1}^{N}\left|\boldsymbol{r}^{(i)}\right|\left|\boldsymbol{v}^{(i)}\right|},
$$

where $\boldsymbol{r}^{(i)}=\boldsymbol{x}^{(i)}-\boldsymbol{x}_{C M}$ is the distance of the $i$-th particle to the center of mass. A (perfect) coherent flock is characterized by $(P, M)=(1,0)$, a (perfect) mill pattern by $(P, M)=$ 

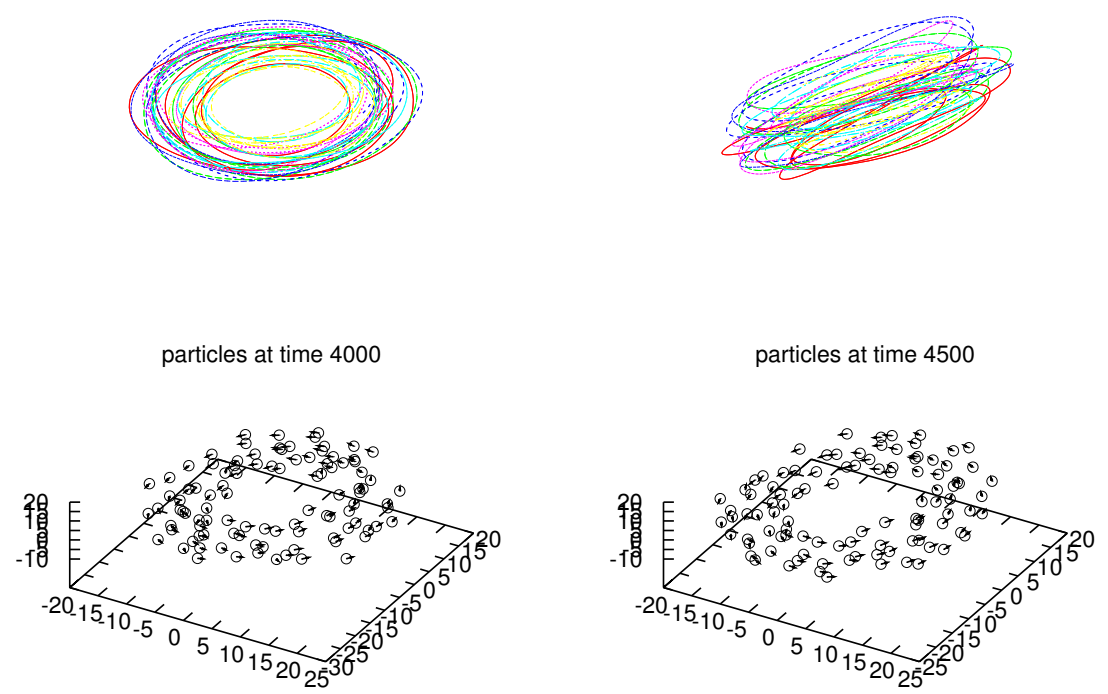

trajectories from time 8000 to time 8500

trajectories from time 8000 to time 8500
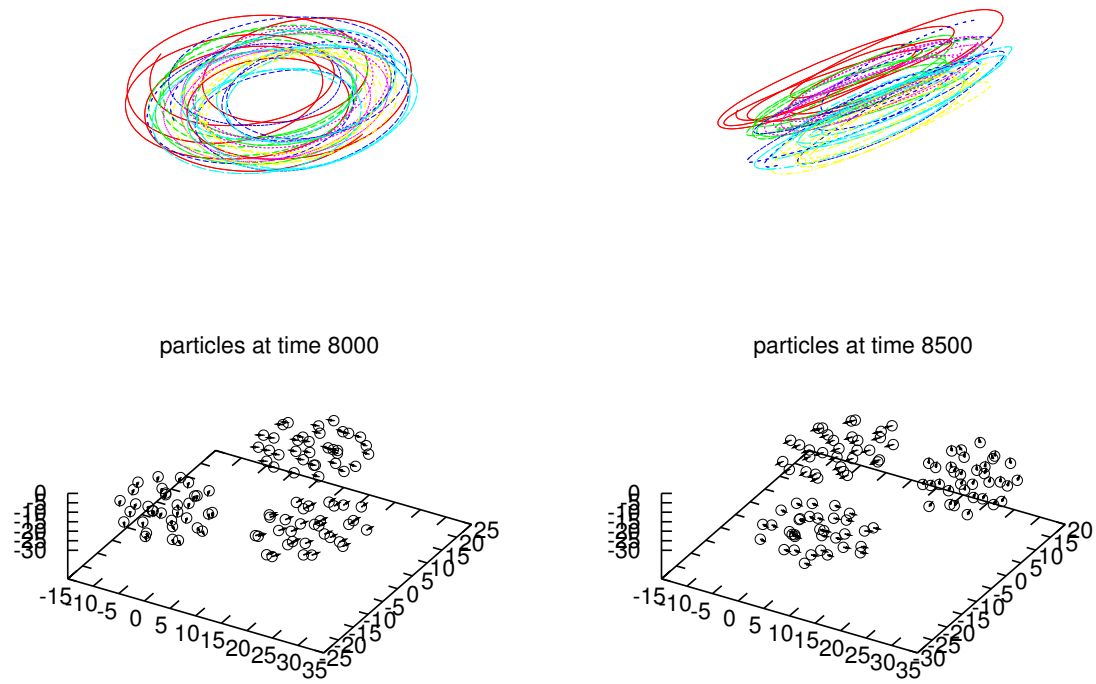

Figure 9: Mills in 3D. Particles turn on an donut shape; after a first stage corresponding to a mill pattern, a rigid-body pattern appears. Parameters are those of case MRK-VII-2 in Table 2. The trajectories are meant those of just some particles, because drawing everyone of them would make the graphic unreadable. 


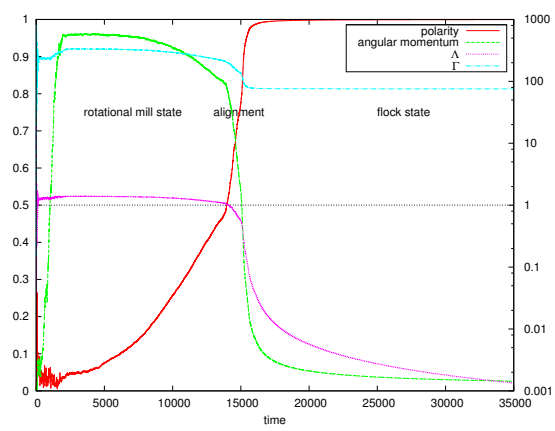

(a) switching dynamics

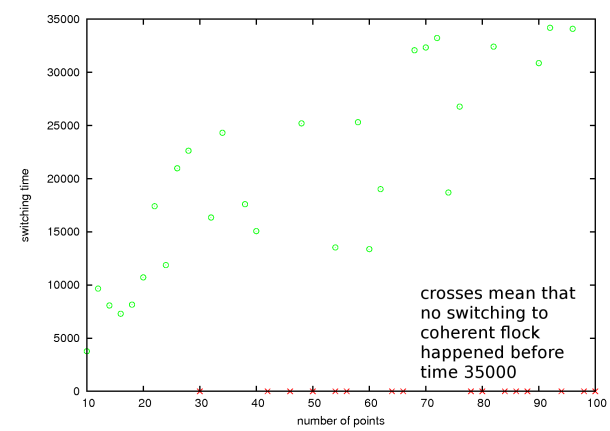

(b) switching time

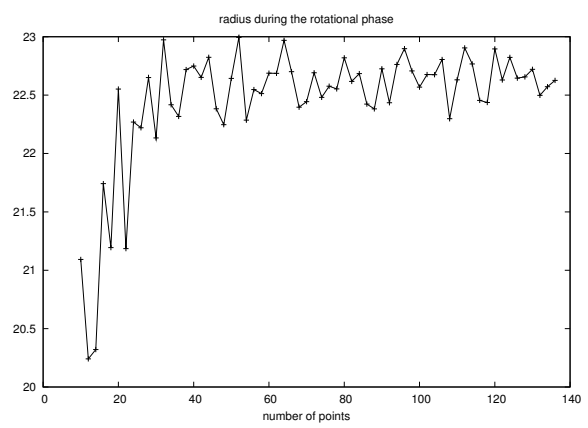

(c) radius

Figure 10: Instability of mills in 3D. Parameters are those of case MRK-VII-2 in Table [2].

$(0,1)$. In Figure 10 (a) we observe how the polarity, after initial oscillations, increases and approaches value 1 , which means that the flock state has been reached. Contextually, both the relative kinetic energy $\Lambda^{N}$ and the relative potential energy $\Gamma^{N}$, defined as

$$
\Lambda^{N}(t):=\frac{1}{2 N^{2}} \sum_{i, j}\left|\boldsymbol{v}^{(i)}(t)-\boldsymbol{v}^{(j)}(t)\right|_{\mathbb{R}^{d}}^{2}, \quad \Gamma^{N}(t):=\frac{1}{2 N^{2}} \sum_{i, j}\left|\boldsymbol{x}^{(i)}(t)-\boldsymbol{x}^{(j)}(t)\right|_{\mathbb{R}^{d}}^{2},
$$

decrease, which means that we are reaching a lower energy level. As the number of particles increases, the switching time from mill to flock increases, probably because the particles encounter more and more difficulties to agree an alignment direction. Numerical experiments (Figure [0 (c)) suggest that the radius of the particle cloud does not seem to depend on the number of agents at all, as seen in 2D in [34].

Shape of the crystal lattice. - An interesting question arises about the organization of the particles in the crystal lattice. In [34] the authors show that the structure is hexagonal by introducing an order-factor. We believe that this strategy can be extended to the 3D setting. First of all we compute the angles particles $j_{1}$ and $j_{2}$ form with respect to particle 
$i$ :

$$
\begin{aligned}
\mathcal{C}_{j_{1}, j_{2}}^{(i)} & =\frac{\left(x^{\left(j_{1}\right)}-x^{(i)}\right) \cdot\left(x^{\left(j_{2}\right)}-x^{(i)}\right)}{\left|x^{\left(j_{1}\right)}-x^{(i)}\right|_{\mathbb{R}^{3}}\left|x^{\left(j_{2}\right)}-x^{(i)}\right|_{\mathbb{R}^{3}}} \\
\phi_{j_{1}, j_{2}}^{(i)} & =\cos ^{-1}\left(\mathcal{C}_{j_{1}, j_{2}}^{(i)}\right) .
\end{aligned}
$$

Then, we order the neighbors of particles $i$ : we use notation $\mathcal{N}_{j}^{(i)}$ such that

$$
\operatorname{dist}\left(x^{(i)}, x^{\mathcal{N}_{1}^{(i)}}\right) \leq \operatorname{dist}\left(x^{(i)}, x^{\mathcal{N}_{2}^{(i)}}\right) \leq \ldots \leq \operatorname{dist}\left(x^{(i)}, x^{\mathcal{N}_{N-1}^{(i)}}\right)
$$

where, for the sake of clarity, we have meant $\mathcal{N}_{0}^{(i)}=i$. We now compute all the angles between the $\mu$ closest particles:

$$
\begin{aligned}
& \text { for } i=0, \ldots, N-1 \\
& \text { for } j_{1}=1, \ldots, \mu \\
& \text { for } j_{2}=j_{1}+1, \ldots, \mu \\
& \quad \text { compute } \phi_{\mathcal{N}_{j_{1}}^{(i)}, \mathcal{N}_{j_{2}}^{(i)}}^{(i)}
\end{aligned}
$$

so that we are taking into account $N\left(\begin{array}{c}\mu \\ 2\end{array}\right)$ angles for each particle $i$. If the lattice is regular enough, we should be able to observe that these angles concentrate around multiples of some value. In order to study the shape of the lattice, we introduce as in [34] an order-factor:

$$
O_{Q}=\frac{1}{N\left(\begin{array}{c}
\mu \\
2
\end{array}\right)}\left|\sum_{i=0}^{N-1} \sum_{j_{1}=1}^{\mu} \sum_{j_{2}=j_{1}+1}^{\mu} \cos \left(Q \cdot \phi_{\mathcal{N}_{j_{1}}^{(i)}, \mathcal{N}_{j_{2}}^{(i)}}^{(i)}\right)\right|
$$

In Figure $\square$ we see that $O$ is peaked at value 2 for any choice of $\mu$, which means, in our opinion, that the particles try to stay on a simple cubic lattice.

$\boldsymbol{\alpha}_{\text {escape }}$ in the catastrophic regime. - By performing the same computations as for Figure [, we can plot the evolution of the radius and the threshold between swarming states and dispersed states in the catastrophic regime: in Figure ए2 we observe that, unlike the results in [34], $\alpha_{\text {escape }}$ seems to decrease as the number of particles increases, thanks to the $\frac{1}{N}$ rescaling in front of the Morse potential that weakens its effect (see Remark [2.2).

\subsection{Patterns in $\mathbb{R}^{3}$ : typical-time point of view}

In this section we wish to give another point of view on what determines the transient and the asymptotic states for the system: from equation (四), which we recall here

$$
\frac{d \boldsymbol{x}^{(i)}}{d t}=\frac{t_{*}}{t_{k i n}} \boldsymbol{v}^{(i)}, \quad \frac{d \boldsymbol{v}^{(i)}}{d t}=\frac{t_{*}}{t_{f / p}}\left(1-\left|\boldsymbol{v}^{(i)}\right|^{2}\right) \boldsymbol{v}^{(i)}-\frac{t_{*}}{t_{a / r}} \frac{1}{N} \sum_{j \neq i} \nabla_{\boldsymbol{x}}[-W(\boldsymbol{x})+C W(\boldsymbol{x} / \ell)],
$$




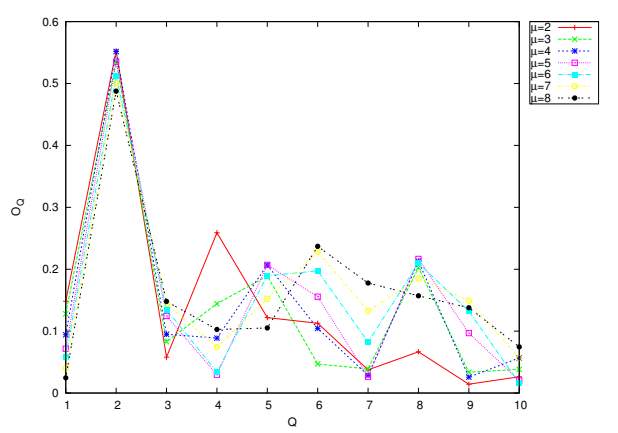

(a) order factor

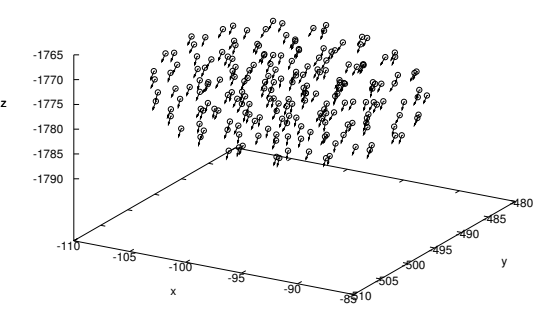

(b) crystalline structure

Figure 11: Shape of the 3D flocks. Parameters are those of case MRK-VII-1 in Table 2, $N=200, \mu=4$. The graphic depicting the order factor $O_{Q}$ shows how particles tend to organize into a simple cubic lattice.

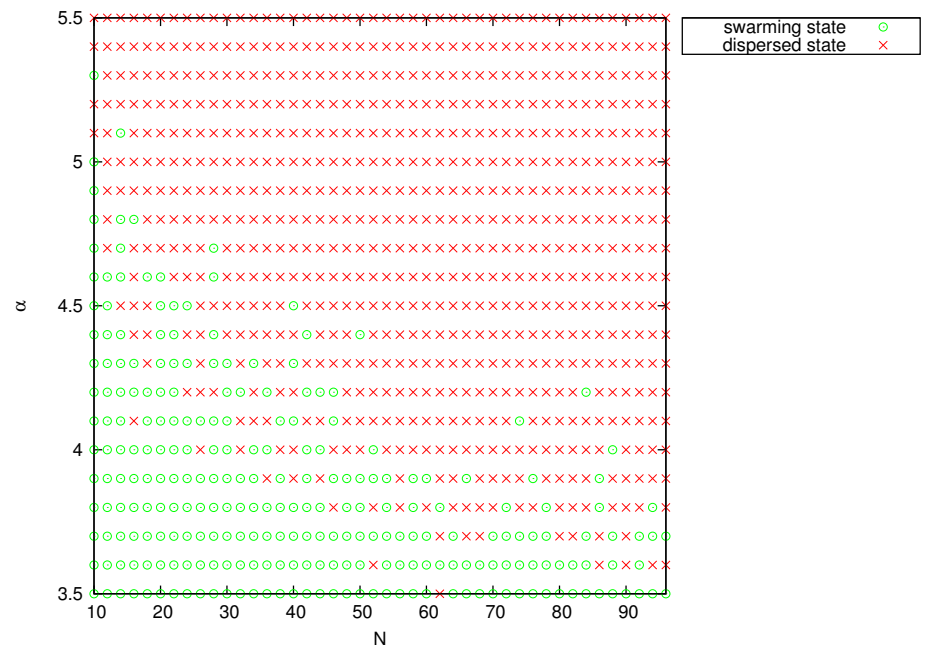

Figure 12: Phase transition. The threshold between swarming states and dispersed states in region VII. Parameters are those of case MRK-VII-3 in Table 2. 
we see that the smaller a typical time $t_{k i n}, t_{f / p}, t_{a / r}$, the more its related phenomenon is dominant. For instance, if $t_{a / r}<t_{p / f}<t_{k i n}$, we expect to observe, along the time evolution of the system, first the phenomenon related to the Morse (attractive/repulsive) potential, then the one related to the Rayleigh (self-propulsion/friction) operator, and asymptotically the one related to the free motion.

\subsubsection{Case MRK: $t_{a / r}<t_{p / f}<t_{k i n}$}

This case, depicted in Figure [3, is the most interesting one. At the beginning, the system oscillates, because the particles try to fix suitable relative distances to minimize the Morse potential. Then, the particles enter a rigid-body state, because $t_{p / f}$ is ranked at the second position. Finally, the system degenerates into a coherent flock, even if conserving some angular momentum.

\subsubsection{Case MKR: $t_{a / r}<t_{k i n}<t_{p / f}$}

This case, depicted in Figure 14, is similar to MRK, the difference being that the particles try to enter a rotational state immediately instead of fixing the relative distances by oscillating around the minima of the Morse potential. Asymptotically, we get a coherent flock.

\subsubsection{Cases KMR $t_{k i n}<t_{a / r}<t_{p / f}$ and KRM $t_{k i n}<t_{p / f}<t_{a / r}$}

In these cases, depicted in Figure 15(a) and Figure 15(b), we get dispersed states, because the free motion term is dominant.

\subsubsection{Cases RMK $t_{p / f}<t_{a / r}<t_{k i n}$ and RKM $t_{p / f}<t_{k i n}<t_{a / r}$}

If the self-propulsion/friction term is dominant, we get a dispersed state, as shown in Figure 16(a) and Figure 16(b). The behavior is qualitatively identical to the cases in which the free motion term is dominant. The self-propulsion/friction term seems to play a role only if the attractive/repulsive term is dominant: it can, in such a situation, act in order to fix the modulus of the velocities; otherwise, the particles are just propelled away, disregarding what the other agents do. 

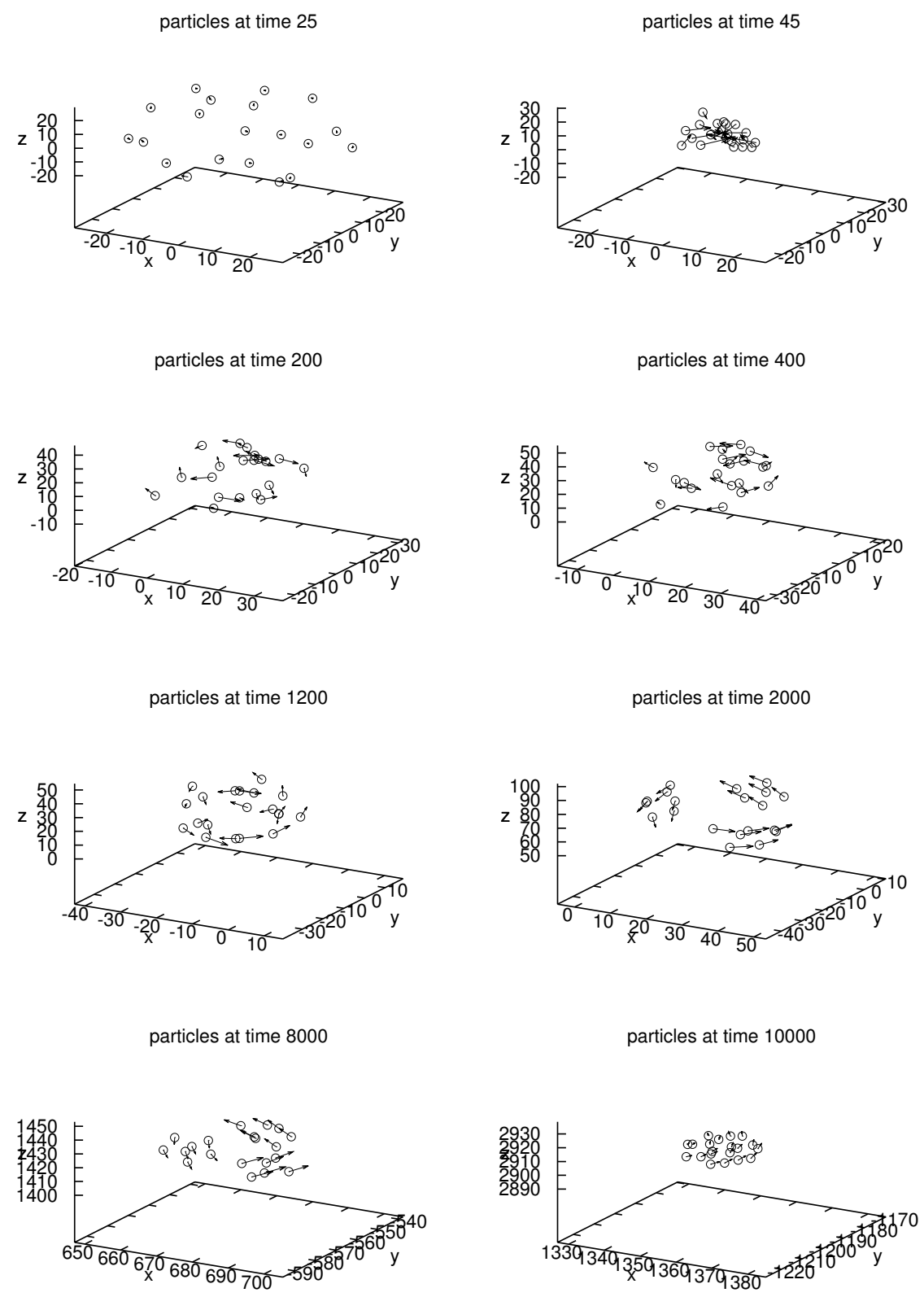

Figure 13: Case MRK. Parameters are those of case MRK-I-2 in Table $\boldsymbol{\nabla}, N=20$. 

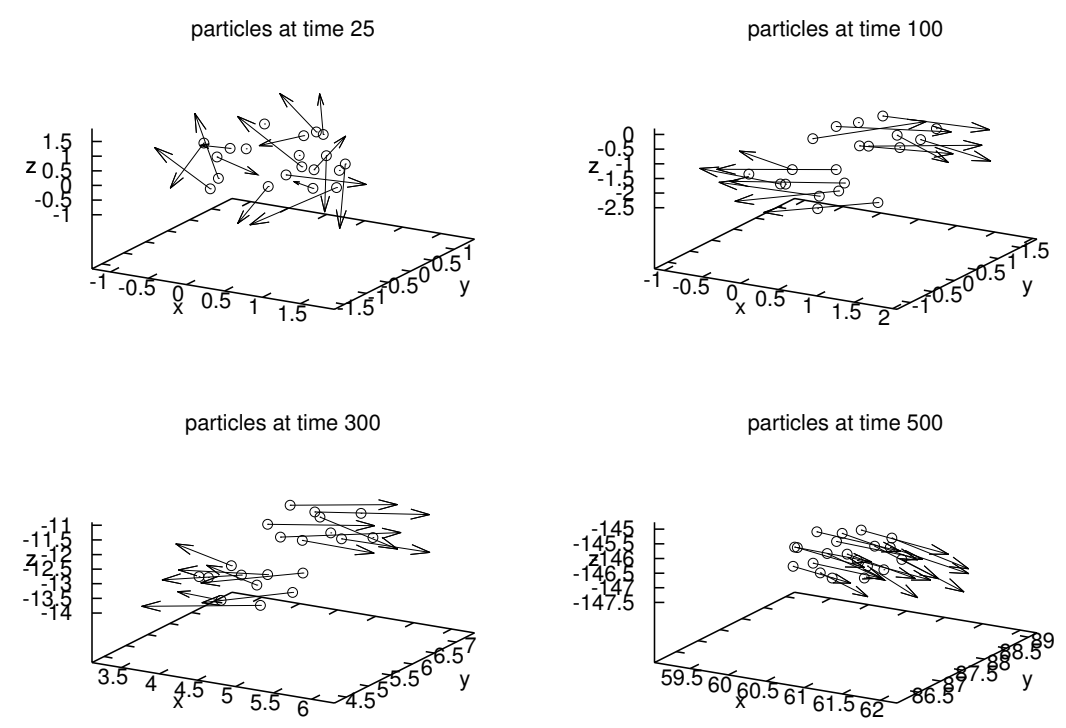

Figure 14: Case MKR. Parameters are those of case MKR-VII in Table [थ, $N=20$. 

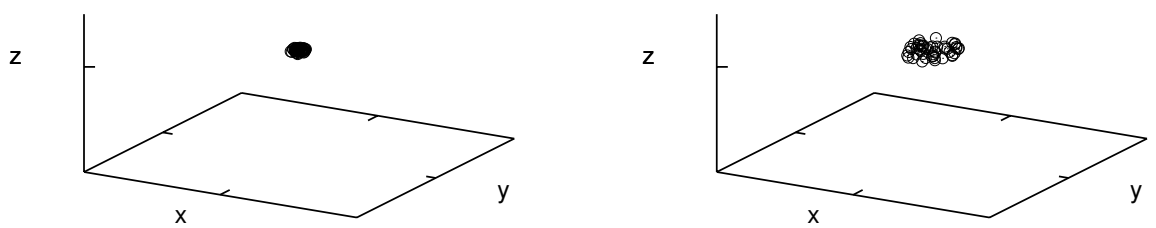

particles at time 30

particles at time 50
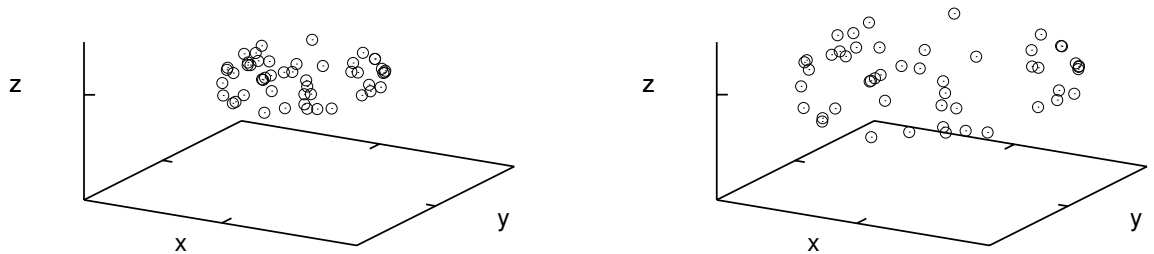

(a) case KMR

particles at time 2.5

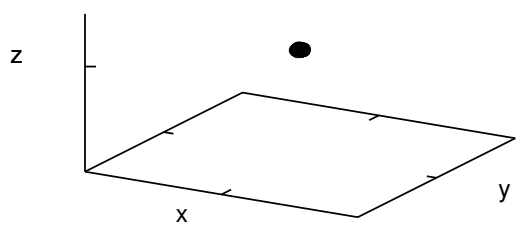

particles at time 30

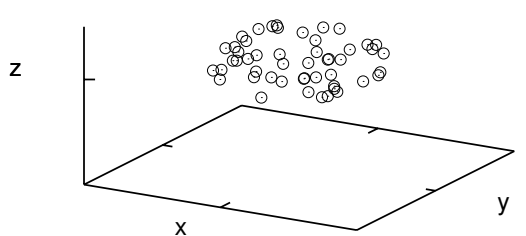

particles at time 10

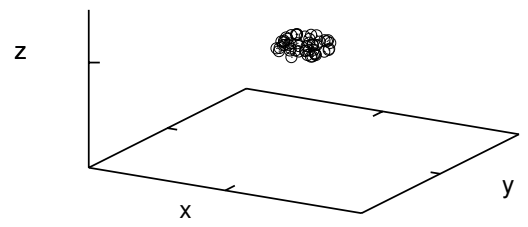

particles at time 50

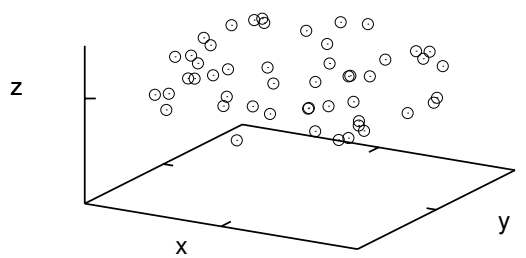

(b) case KRM

Figure 15: Cases KMR and KRM. Parameters are: (a) case KMR-VII in Table 2, $N=50$; (b) case KRM-VII in Table $\square, N=50$. We get a dispersed state. 

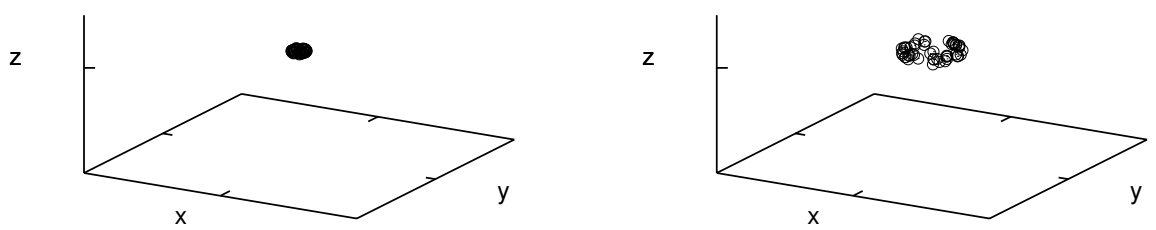

particles at time 30

particles at time 50
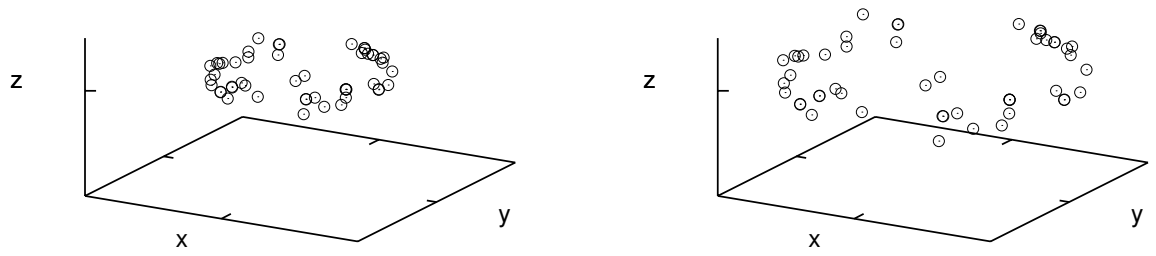

(a) case RMK

particles at time 2.5

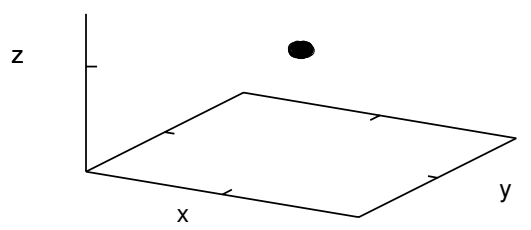

particles at time 30

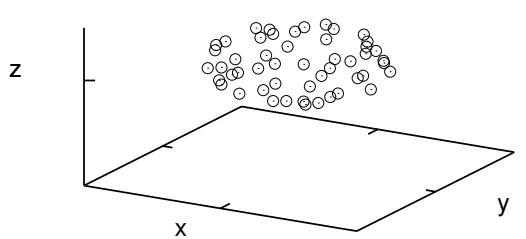

particles at time 10

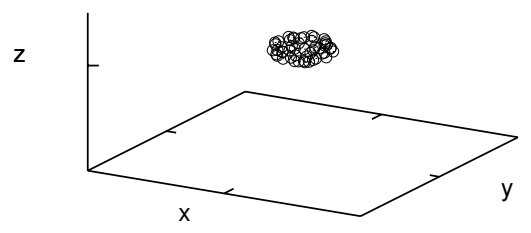

particles at time 50

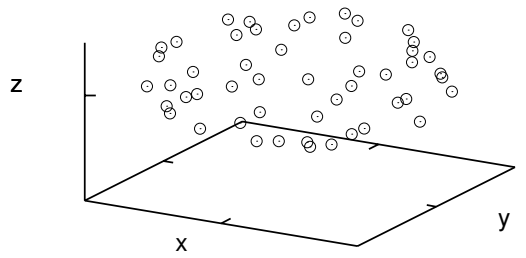

(b) case RKM

Figure 16: Cases RMK and RKM. Parameters are: (a) case RMK-VII in Table $2, N=50$; (b) case RKM-VII in Table $\square, N=50$. We get a dispersed state. 


\section{The continuum model}

\subsection{General guide rules}

In this section we want to study numerically the solutions to the $1 \mathrm{D}$ kinetic version of the model,

$$
\partial_{t} f+v \cdot \partial_{x} f+\partial_{v}\left[\left(\alpha-\beta v^{2}\right) v f\right]-\partial_{v}\left[\left(\partial_{x} U * \rho\right) f\right]=0, \quad t>0, x \in[0,2 \pi R), v \in \mathbb{R}
$$

with periodic boundary conditions, modeling the behavior of the school on a ring of fixed radius $R$ ( $x$ should be thought of as an arc length).

Indeed, it was shown in the particle simulations that, for example for MRK-VII-2, the particles tend to turn on a donut shape. It therefore seems adequate to study the long-time behavior of the distribution of particles on circles.

According to the derivation performed in [36], the continuum model corresponding to our dimensionless setting (3)-(5) is

$$
\partial_{t} f+\frac{t_{*}}{t_{k i n}} v \cdot \partial_{x} f+\frac{t_{*}}{t_{f / p}} \partial_{v}\left[\left(1-v^{2}\right) v f\right]-\frac{t_{*}}{t_{a / r}} \partial_{v}\left[\left(\partial_{x}\left(-e^{-|x|^{p}}+C e^{-\frac{|x|^{p}}{\ell^{p}}}\right) * \rho\right) f\right]=0,
$$

where the (short range) convolution should be understood in the $1 \mathrm{D}$ torus. Here, $f \in$ $\mathcal{C}\left([0,+\infty), \mathcal{P}^{1}(\Omega)\right)$ is provided with initial condition $f(0, \cdot, \cdot) \in \mathcal{P}^{1}(\Omega)$, periodic boundary conditions for the $x$-domain and Dirichlet boundary conditions for the $v$-domain, which should be an acceptable assumption in the discretization since we expect that no particles should be there unless the size of the domain is wrongly chosen. We use here a Strang splitting scheme, solving the advection part with an upwind finite difference scheme in $x$, the attraction/repulsion part with a finite difference scheme in $v$ and the friction/autopropulsion part with a conservative semi-lagrangian scheme (see [44]).

Before going into more details in this respect, let us precise some of the continuous properties that we want the scheme to respect numerically:

1. For the $v$-derivatives (friction+convolution), we want the scheme to conserve the mean value in $v$. The way to do that is to adapt the boundary terms of the finite differences formulae and to adopt a very good reconstruction scheme (PFC3) for the conservative semi-lagrangian scheme.

2. We want the total mass in $(x, v)$ to be conserved, so we have to be careful with the convolution term. In 1D, we handle it by the use of Laguerre quadrature points. We choose here to make all the simulations in the case $p=1$, although the case $p=2$ can be treated in a very similar manner with Hermite quadrature points.

3. We want the scheme to respect the maximum principle that ensures the $L^{\infty}$ stability for the advection and convolution parts. This imposes a CFL type condition that links $\Delta t$ to $\Delta x$ for the advection part, to $\Delta v$ for the convolution part. Moreover, the use of the semi-lagrangian scheme links $\Delta t$ to $v_{\max }$ (see the extensive computations in $\mathbb{A}$ and in particular ([प, )). 


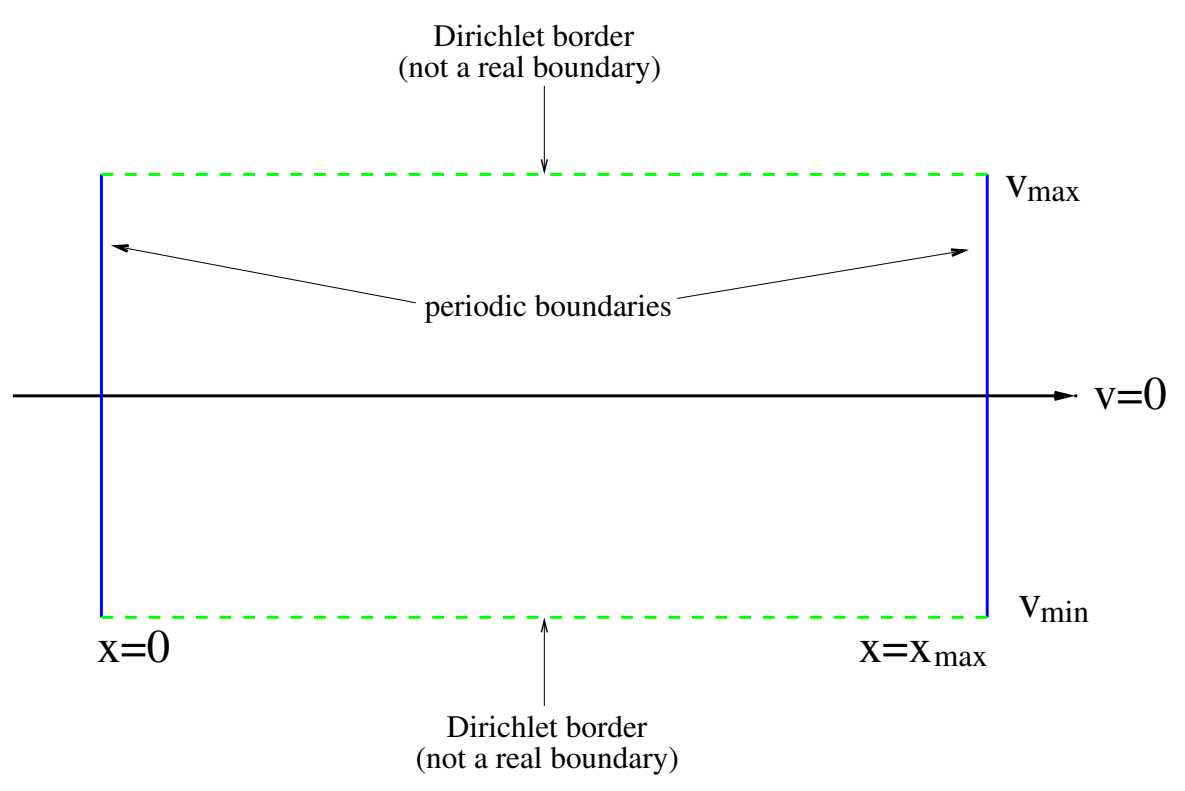

Figure 17: The boundary conditions for the 1D kinetic problem.

\subsection{Numerical scheme for the kinetic model}

We develop a scheme for the $1 \mathrm{D}$ case. The unbounded velocity domain $\mathbb{R}$ needs to be cut for computational purposes; therefore, we shall use [ $\left.-v_{\max }, v_{\max }\right]$.

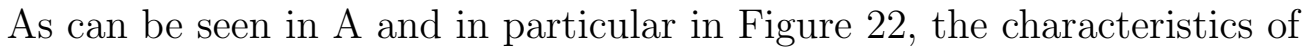

$$
\partial_{t} f+\partial_{v}\left(\left(1-v^{2}\right) v f\right)=0
$$

are very steep and concentrate around \pm 1 exponentially fast: therefore, we will choose $v_{\max }=3$ for all the numerical simulations, assuming the initial datum is compactly supported in the interval $[-3,3]$. As for the boundary conditions, we use periodicity for the $x$-domain: for $L=2 \pi R$

$$
f\left(t^{n},-L, v\right)=f\left(t^{n}, x_{\max }-L, v\right), \quad f\left(t^{n}, x_{\max }+L, v\right)=f\left(t^{n}, L, v\right),
$$

while we use Dirichlet boundary conditions for the $v$-domain:

$$
f\left(t^{n}\right)=0 \text { for } v \in\left\{-v_{\max }, v_{\max }\right\} ;
$$

the situation is sketched in Figure ㅁ].

Discretization. - Both the $x$-space and the $v$-space are meshed with uniform grids:

$$
\begin{aligned}
x_{i}=(i-1) \Delta x, & \text { for } i=1, \ldots, N_{x}, \quad \Delta x=\frac{x_{\max }}{N_{x}}, \\
v_{j}=-v_{\max }+(j-1) \Delta v, & \text { for } j=1, \ldots, N_{v}, \quad \Delta v=\frac{2 v_{\max }}{N_{v}},
\end{aligned}
$$

while we advance in time through a second-order splitting scheme [45, 46]: 
Step (i). Solve for a $\Delta t / 2$-time step

$$
\partial_{t} f-\frac{t_{*}}{t_{a / r}} \partial_{v}\left[\left(\partial_{x}\left(-e^{-|x|}+C e^{-\left|\frac{x}{\ell}\right|}\right) * \rho\right) f\right]=0 .
$$

Step (ii). Solve for a $\Delta t / 2$-time step

$$
\partial_{t} f+\frac{t_{*}}{t_{k i n}} v \cdot \partial_{x} f=0 .
$$

Step (iii). Solve for a $\Delta t$-time step

$$
\partial_{t} f+\frac{t_{*}}{t_{f / p}} \partial_{v}\left[\left(1-|v|^{2}\right) v f\right]=0 .
$$

Step (iv). Same as Step (ii).

Step (v). Same as Step (i).

Of course, a CFL condition is needed for the scheme to be stable (see Subsection [.]) and has to be updated at each time step:

$$
\Delta t \leq \mathrm{CFL} \cdot \min \left\{\frac{t_{k i n}}{t_{*}} \frac{\Delta x}{\|v\|_{\infty}}, \frac{t_{f / p}}{2 t_{*}} \log \left(\frac{v_{\max }^{2}}{\left(v_{\max }^{2}-1\right)}\right), \frac{t_{a / r}}{t_{*}} \frac{\Delta v}{\left\|\partial_{x}\left(-e^{-|x|}+C e^{-\frac{|x|}{\ell}}\right) * \rho\right\|_{\infty}}\right\} .
$$

For the sake of simplicity, we detail now the Lie version of the splitting scheme.

Solving Step(i). - From the properties of the convolution operator, we have

$$
\partial_{x}\left[\left(-e^{-|x|}+C e^{-\left|\frac{x}{\ell}\right|}\right) * \rho\right]=-\partial_{x} e^{-|x|} * \rho+C \partial_{x} e^{-\left|\frac{x}{\ell}\right|} * \rho .
$$

Straightforward computations give, for the convolution terms:

$$
\partial_{x} e^{-\left|\frac{x}{\ell}\right|} * \rho=\int_{0}^{L} e^{-y} \rho(x+\ell y) \mathrm{d} y-\int_{0}^{L} e^{-y} \rho(x-\ell y) \mathrm{d} y,
$$

which, injected into (8), gives

$$
\begin{aligned}
\partial_{x}\left[\left(-e^{-|x|}+C e^{-\frac{|x|}{\ell}}\right) * \rho\right]= & -\int_{0}^{L} e^{-y} \rho(x+y) \mathrm{d} y+\int_{0}^{L} e^{-y} \rho(x-y) \mathrm{d} y \\
& +C \int_{0}^{L} e^{-y} \rho(x+\ell y) \mathrm{d} y-C \int_{0}^{L} e^{-y} \rho(x-\ell y) \mathrm{d} y .
\end{aligned}
$$

We now use Laguerre collocation points [47]

$$
\int_{x}^{L} e^{-|x|} F(x) \mathrm{d} x \approx \sum_{i=1}^{K} \omega_{i} F\left(X_{i}\right)
$$


where $K$ is the order of precision of the method, the $\left\{\omega_{i}\right\}_{i}$ are weights and the $\left\{X_{i}\right\}_{i}$ are quadrature points. Using this formula, we obtain

$$
\begin{aligned}
a_{i}=\partial_{x}\left[\left(-e^{-|x|}+C e^{-\frac{|x|}{\ell}}\right) * \rho\right]\left(x_{i}\right) \approx & -\sum_{r=1}^{K} \omega_{r} \rho\left(x+X_{r}\right)+\sum_{r=1}^{K} \omega_{r} \rho\left(x-X_{r}\right) \\
& +C \sum_{r=1}^{K} \omega_{r} \rho\left(x+\ell X_{r}\right)-C \sum_{r=1}^{K} \omega_{r} \rho\left(x-\ell X_{r}\right),
\end{aligned}
$$

where the density $\rho(x)$ is extended with zero-values outside the domain $\left[x_{\min }, x_{\max }\right]$. Once the convolution has been evaluated, an upwind scheme is used for the derivatives, and an explicit Euler to advance in time:

$$
\frac{f_{i, j}^{n+1 / 3}-f_{i, j}^{n}}{\Delta t}-\frac{t_{*}}{t_{a / r}} \times\left\{\begin{array}{ll}
a_{i} \frac{f_{i, j+1}^{n}-f_{i, j}^{n}}{\Delta v} & \text { if } a_{i}>0 \\
a_{i} \frac{f_{i, j}^{n}-f_{i, j-1}^{n}}{\Delta v} & \text { if } a_{i}<0
\end{array}=0 .\right.
$$

Solving Step(ii). - An upwind scheme is used for the derivatives, and an explicit Euler to advance in time:

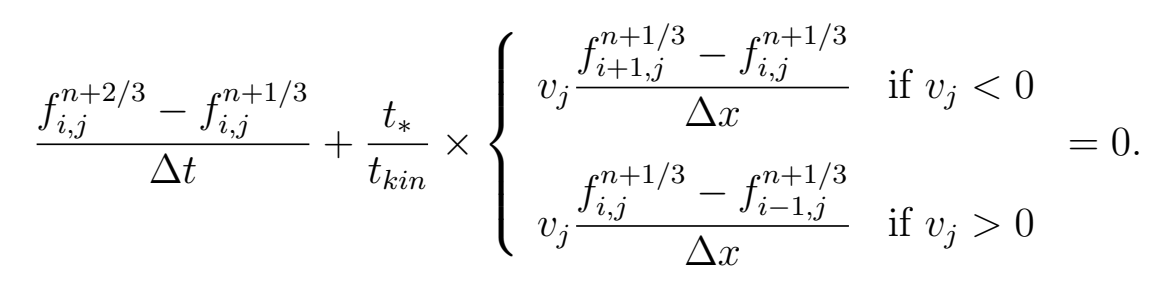

Solving Step(iii). - To solve

$$
\partial_{t} f+\frac{t_{*}}{t_{f / p}} \partial_{v}\left[\left(1-v^{2}\right) v f\right]=0
$$

a conservative semi-lagrangian scheme with PFC3 reconstruction (see [44] and 太A) is used.

\subsubsection{Properties of the scheme}

This simple explicit scheme used to advance in time is no better than one-order accurate, since we chose upwind schemes for Steps (i) and (ii), nevertheless it possesses some useful properties:

- Mass conservation: each step of the splitting schemes conserves the mean value in $v$ and the total mass.

- Maximum principle: the maximum is preserved in the advection parts (Step (i) and (ii) both in the continuous and the discrete setting (under a well-chosen CFL condition)), Step (ii) allowing $f$ to concentrate in $v$ around the asymptotic limits 
of the characteristics \pm 1 . Note that, even if we know how to solve the linear part of the equation $(\mathrm{K}+\mathrm{R})$, we chose to split the operator into two parts to reduce the computational costs.

- Consistency: the scheme is consistent with Equation (7) by construction and show to be little diffusive.

- Direction conservation: we observed numerically and systematically that the mass of particles initially going leftwards (resp. rightwards) is conserved in time.

\subsection{Numerical results}

The following results were obtained with $N_{x}=102$ and $N_{v}=33$. The number of GaussLaguerre quadrature points for the convolution is 4 . For an initial datum with clumps, we represent here the discrete $f$ at $t=0, t=500$ and $t=4000$ in the phase space (see e.g. Figure 18(b]) and the densities $\rho_{+}=\int_{v>0} f \mathrm{~d} v$ and $\rho_{-}=\int_{v<0} f \mathrm{~d} v$ the masses of which are conserved in time. In order to give an extensive, but compact view, we show $\rho_{+}$as a positive quantity and $\rho_{-}$as a negative quantity on the same periodic graph (see e.g. Figure 19(a)). Moreover, the simulations are presented in a dimensionless $x$ and $v$ setting, but the time $T \in\{500,4000\}$ is the physical one to ensure a good comparison.

Let us comment briefly these results:

- General comment. As predicted, asymptotically, the particles divide into two groups with opposite velocities of modulus 1, that is the selfpropulsion/friction effect is well reproduced by the scheme.

- KRM-VII. The effect of the kinetic part is clearly predominant and uniformizes the initial group on the whole torus.

- MKR. Particles tend to occupy the whole torus asymptotically if the parameters correspond to regions I and II (note that these parameters are not biologically relevant since $C<1)$. It is therefore very interesting to observe that, in region VI, even if the $\mathrm{R}$ effect is the last to kick in, the velocities of the particles are well fixed around \pm 1 rather quickly. The particles will take a very long time to occupy the whole torus. Note that, at $t=4000$, the clumps have already turned around the whole torus (the large clump moves leftwards). Asymptotically, rings will be formed, consistently with the particle simulations (see Figure $\mathbf{\theta}$ ).

- MRK-VII. The initial clumps get regularized very slowly, due to the inherent diffusion of the scheme but do not move in space. It is expected since the configuration lies in region VII: this result is consistent with the stable rigid-body behavior that can be observed in Figure 9 .

- KMR-VII. The difference in behavior (uniform wrt clump) with the MRK-VII behavior lies in the large ratio of $t_{k i n}$. 
- KMR. We observe that, consistently with the particle simulations, the particles are uniformly occupying the torus asymptotically, since the kinetic term is dominant.

- For RKM and RMK we do not show any simulation due to the computational cost of arriving to the same final time as in the other cases.

We stress the point that the 1D observations are deriving from a "well-prepared" initial conditions which corresponds to a supposed 2D or 3D asymptotic state. Therefore, what is reflected in 1D is mainly the order of the dominant effects. Nonetheless, these results will prove very useful to validate the $2 \mathrm{D}$ code.

\section{Conclusions and future plans}

In this paper, we have implemented a 3D solver at particle level and a $1 \mathrm{D}$ solver on a ring (i.e. with periodic boundaries) at kinetic level. We have given a key to interpret the qualitative behavior of the system based on ranking the typical times for the free motion, the self-propulsion/friction term and the attractive/repulsive term. We have performed a numerical analysis on the 3D particle system, to show the emergence of several different patterns (clumps, rings, mills, rigid-body rotations, flocks, dispersed states), depending on the H-stability region or on the hierarchy of typical times. We recover many of the qualitative behaviors observed in the 2D case [34], but there are two important differences within the two models:

- The rescaling weakens the attraction/repulsion effect of the potential. Therefore the system encounters increasing difficulties to prevent particles from dispersing both in regions VI and VII.

- In the case of crystalline structures the shape of the lattice is cubic instead of hexagonal.

We have then designed a numerical scheme for the kinetic simulations based on a secondorder Strang-splitting procedure, which has two good properties: it is conservative and, moreover, the maximum principle is respected except by the self-propulsion/friction part. Still, several things need to be developed in the future:

- We are implementing a 2D solver at kinetic level: it must be completed, debugged, and tested to show which patterns we can recover, in comparison to the $2 \mathrm{D}$ particle simulations.

- We want to show with numerical evidence that the kinetic model is the $N \rightarrow \infty$ limit of the particle model, by studying the convergence in the sense of the Wasserstein measure.

- We want to investigate with more detail the reasons that make the mills degenerate into coherent flocks after a certain transient time. 


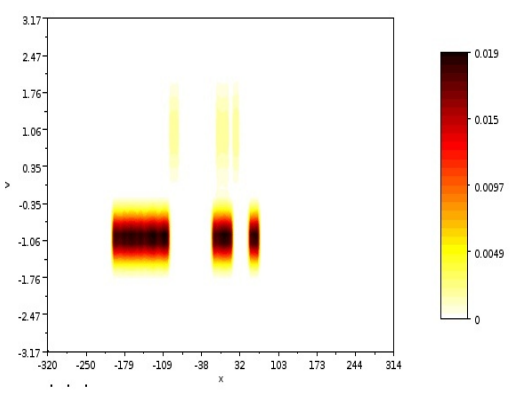

(a) Initial condition

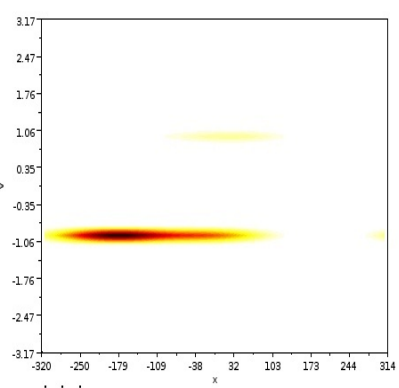

(b) Case MKR-I

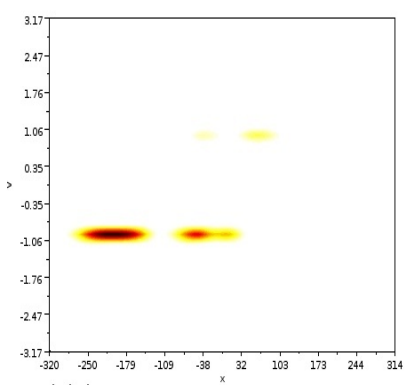

(d) Case MKR-VI-1

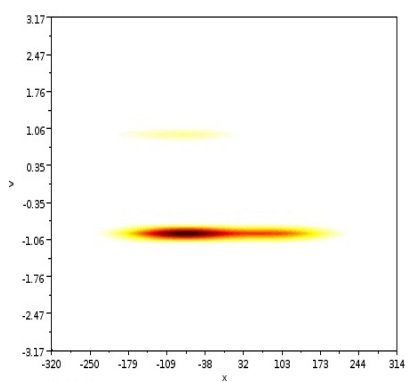

(f) Case KMR-VII
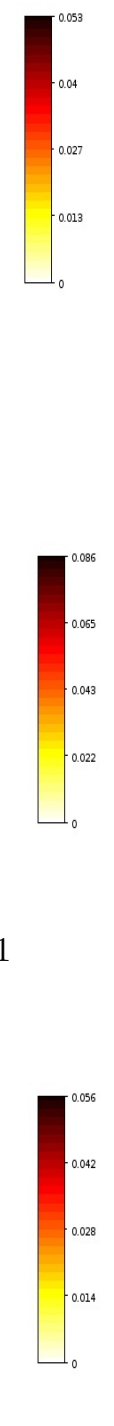

Figure 18: Particle distribution $f$ at time $t=0$ (a) and $t=500$ 


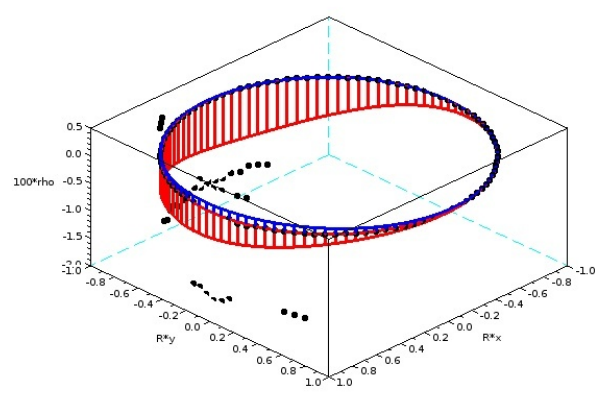

(a) Case MKR-I

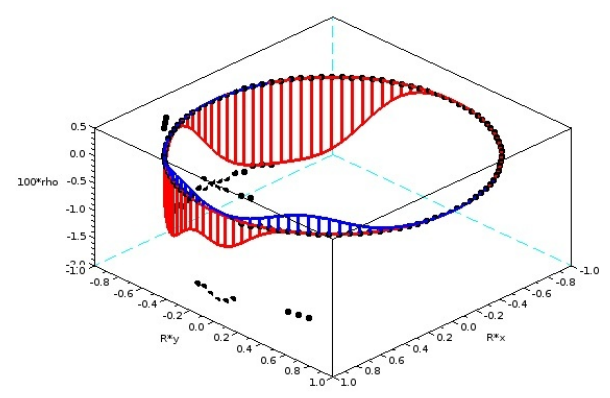

(c) Case MKR-VI-1

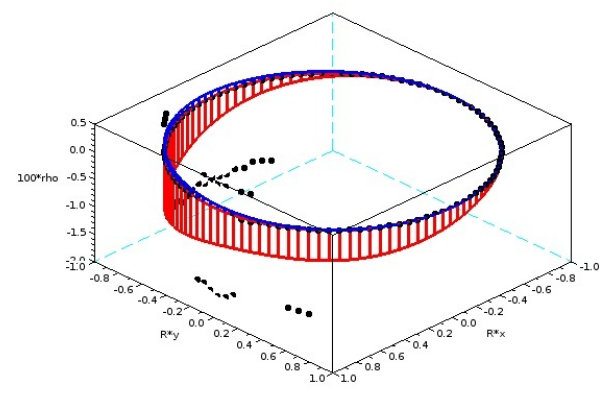

(e) Case KMR-VII

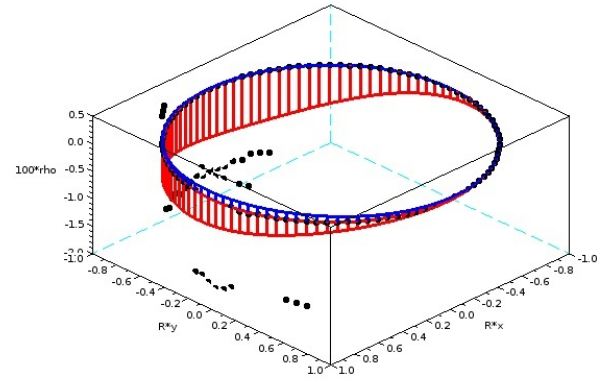

(b) Case MKR-II

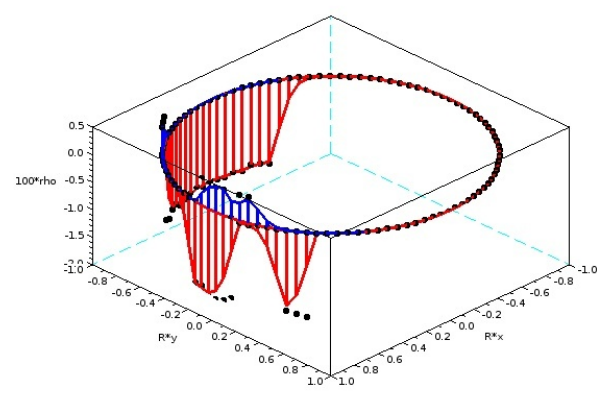

(d) Case MRK-VII-2

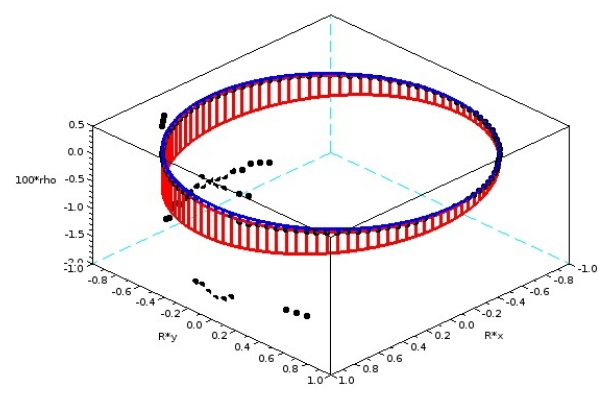

(f) Case KRM-VII

Figure 19: Macroscopic densities $\rho_{+}$(above 0 ) and $\rho_{-}$(below 0 ) at time $t=0$ (square marks) and $t=500$ 


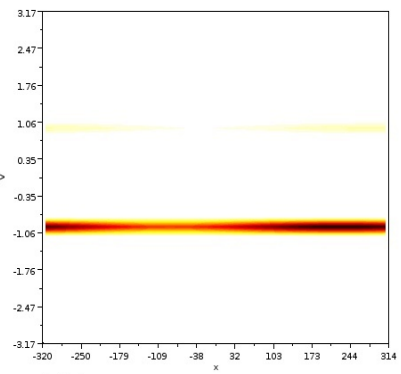

(a) Case MKR-I

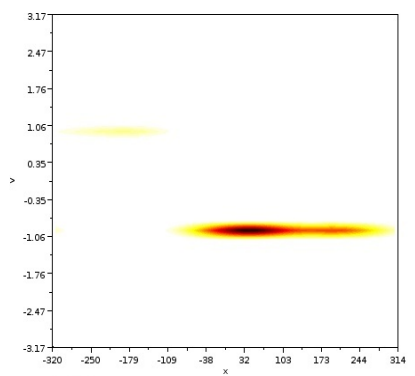

(c) Case MKR-VI-1

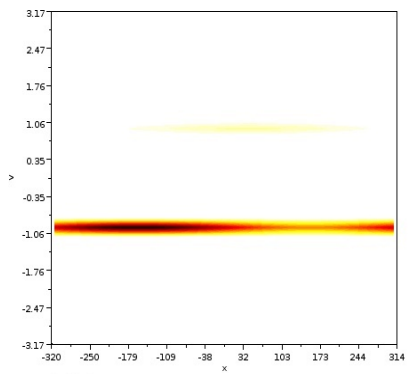

(e) Case KMR-VII
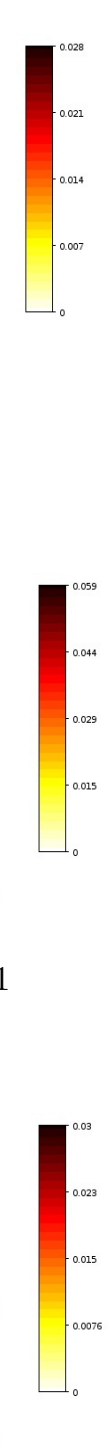

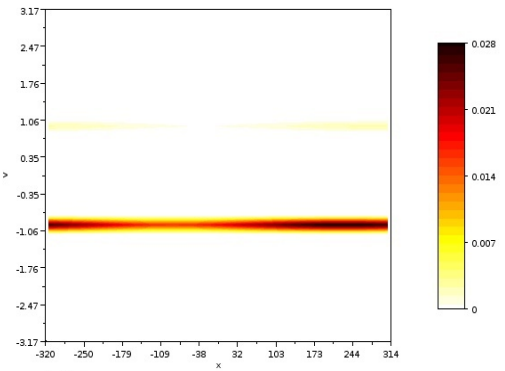

(b) Case MKR-II

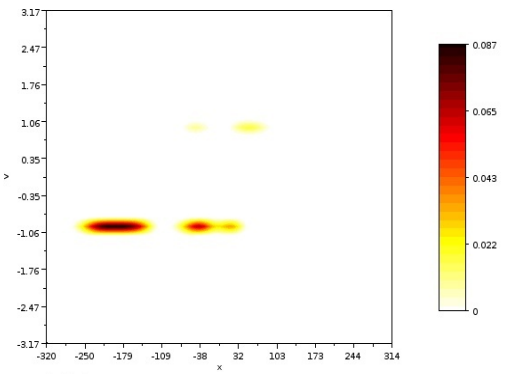

(d) Case MRK-VII-2

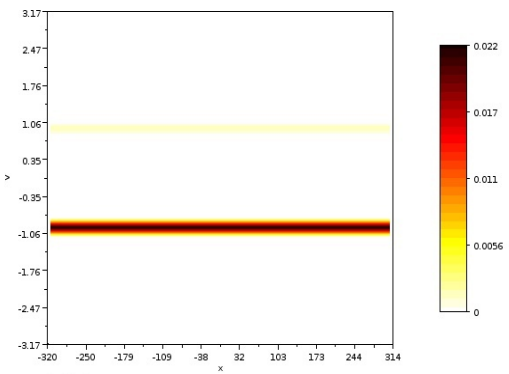

(f) Case KRM-VII

Figure 20: Particle distribution $f$ at time $t=4000$ 


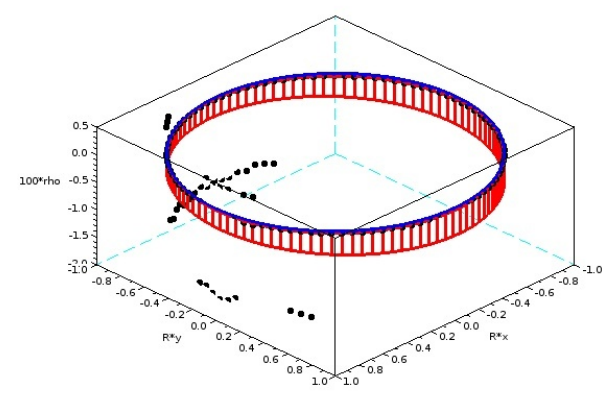

(a) Case MKR-I

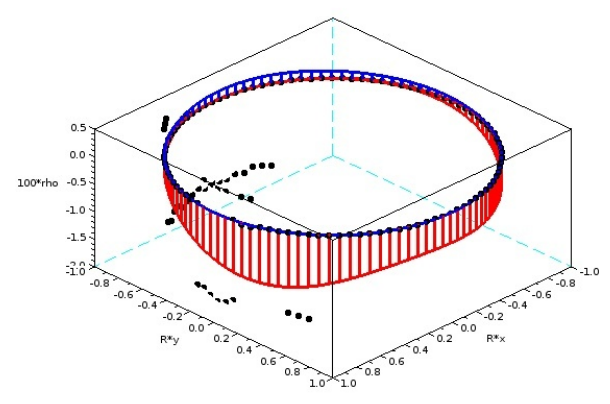

(c) Case MKR-VI-1

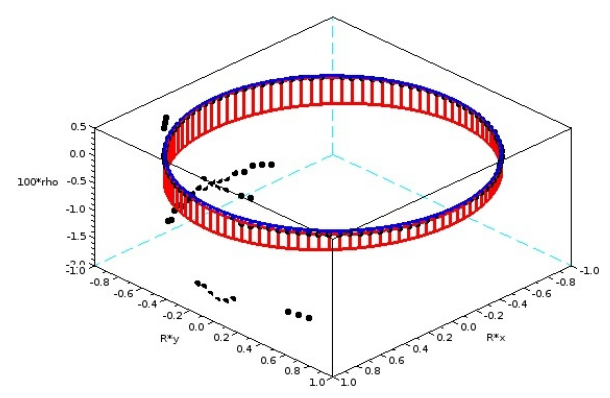

(e) Case KMR-VII

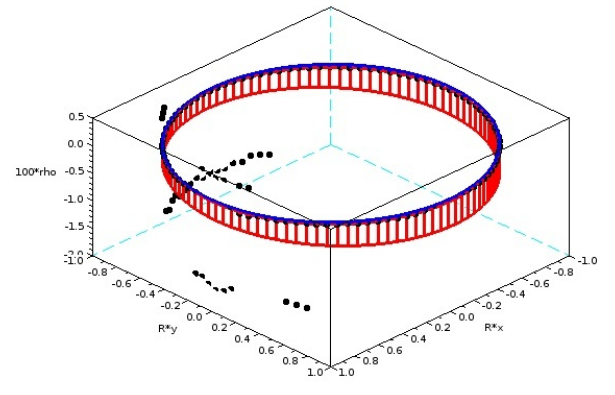

(b) Case MKR-II

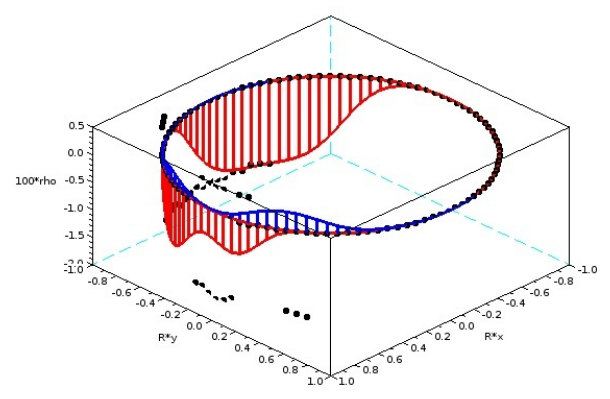

(d) Case MRK-VII-2

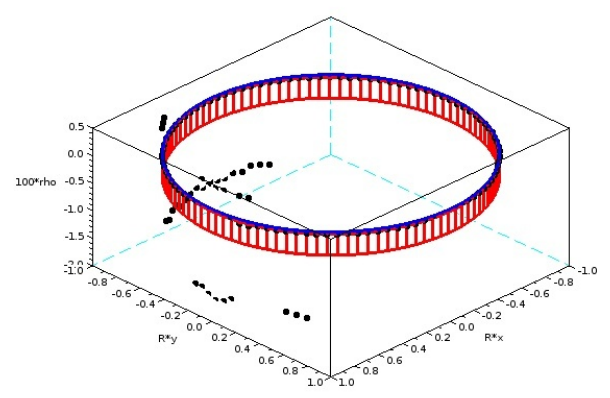

(f) Case KRM-VII

Figure 21: Macroscopic densities $\rho_{+}$(above 0$)$ and $\rho_{-}$(below 0$)$ at $t=4000$ 
- The particle code allows for a straightforward parallelization. The kinetic solver can also be parallelized, in order to reduce the computational cost of the $2 \mathrm{D}$ (4D in the phase space) or 3D (6D in the phase space) models.

\section{Acknowledgments}

The authors would like to thank Professor José Antonio Carrillo for encouraging them and for many fruitful and interesting discussions and Professor Andrea L. Bertozzi for her enlightening comments. PL was partly supported by the University of Lille 1, the EPI SIMPAF, INRIA Lille Nord Europe and the MAS Lab. at Ecole Centrale Paris. JR was partially supported by the FSMP, CBDif, ARO MURI grant W911NF-11-1-0332, NSF grant EFRI1024765 and NSF grant DMS-0907931. FV acknowledges the MINECO project MTM201122741. 


\section{A Characteristics}

Let us detail the full computations leading to the explicit resolution of the linear part of the equation, that involves the advection and friction terms in $d$ dimensions. We want to solve

$$
\begin{array}{cl}
\partial_{t} f+v \cdot \nabla_{x} f+\nabla_{v}\left(\left(\alpha-\beta\|v\|^{2}\right) v f\right)=0, & t>0, x, v \in \mathbb{R}^{d} \\
f(0, x, v)=f^{0}(x, v), & x, v \in \mathbb{R}^{d} .
\end{array}
$$

Let $\left(x^{0}, v^{0}\right) \in \mathbb{R}^{d} \times \mathbb{R}^{d}$. The characteristic curve $t \mapsto(X(t), V(t))$ starting at $(X(0), V(0))=$ $\left(x^{0}, v^{0}\right)$ solves the differential system

$$
\begin{aligned}
\dot{X} & =V, \\
\dot{V} & =\left(\alpha-\beta\|V\|^{2}\right) V=: G(V), \\
X(0) & =x^{0} \\
V(0) & =v^{0} .
\end{aligned}
$$

Let us solve at first the Cauchy problem for $Z:=\|V\|^{2}$ :

$$
\begin{aligned}
\frac{\dot{Z}}{2} & =(\alpha-\beta Z) Z \\
Z(0) & =\left\|v^{0}\right\|^{2}
\end{aligned}
$$

Let us denote $w:=\sqrt{\alpha / \beta}$. We note that 0 and $w^{2}$ are the two stationary points and that 0 is attractive whereas $w^{2}$ is repulsive. The solution of the Cauchy problem is thus defined globally. For $\left\|v^{0}\right\|^{2} \in \mathbb{R}^{+} \backslash\left\{0, w^{2}\right\}$, the solution of this problem is

$$
Z: t \mapsto \frac{\left\|v^{0}\right\|^{2} e^{2 \alpha t}}{1+\left(e^{2 \alpha t}-1\right) \frac{\left\|v^{0}\right\|^{2}}{w^{2}}}
$$

so that we note at once that $Z(t)$ converges towards $w^{2}$ as $t$ goes to $+\infty$. To compute $V$ and $X$, we now need to solve the ODEs on each component. If we define:

$$
S_{\alpha, w, v^{0}}: t \mapsto 1+\left(e^{2 \alpha t}-1\right) \frac{\left\|v^{0}\right\|^{2}}{w^{2}}
$$

- for $V$, we get

$$
\forall j \in\{1, \ldots, d\}, \frac{\dot{V}_{j}}{V_{j}}=\alpha-\beta Z,
$$

so that $V: t \mapsto \frac{e^{\alpha t}}{\sqrt{S_{\alpha, w, v^{0}}(t)}} v^{0}$ and $\lim _{+\infty} V=w \frac{v^{0}}{\left\|v^{0}\right\|}$;

- and for $X$, we get

$$
\forall j \in\{1, \ldots, d\}, \dot{X}_{j}=V_{j},
$$

and $X: t \mapsto x^{0}+\frac{1}{\alpha} \log \left(\frac{\left\|v^{0}\right\| e^{\alpha t}+w \sqrt{S_{\alpha, w, v^{0}}(t)}}{\left\|v^{0}\right\|+w}\right) \frac{w}{\left\|v^{0}\right\|} v^{0}$. 
Along these characteristics $t \mapsto(t, X(t), V(t)), V^{2}=(\alpha-\dot{V} / V) / \beta$ and, if $f^{0}\left(x^{0}, v^{0}\right) \neq 0$ the solution $g: t \mapsto f(t, X(t), V(t))$ satisfies

$$
\dot{g}=(d \alpha-(d+2) \beta Z) g
$$

so that

$$
g: t \mapsto e^{-d \alpha t} f^{0}\left(x^{0}, v^{0}\right)\left(1+\left(e^{2 \alpha t}-1\right) \frac{\left\|v^{0}\right\|^{2}}{w^{2}}\right)^{1+d / 2} .
$$

So finally $f$ should concentrate in $v$ around $\|v\|=w$, as we can see in Figure [2].

A characteristic curve $s \mapsto(s, \tilde{X}(s), \tilde{V}(s))$ which passes through $(t, x, v) \in[0,+\infty) \times d \mathbb{R} \times$ $\mathbb{R}^{d}$ is defined if and only if $\left(\|v\|^{2} \leq \alpha / \beta\right)$ or $\left(\|v\|^{2}>\alpha / \beta\right.$ and $\left.2 \alpha t<\log \left(\beta\|v\|^{2} /\left(\beta\|v\|^{2}-\alpha\right)\right)\right)$ and, if it exists, it has the following expression $\forall s \in[0, t]$, :

$$
\begin{aligned}
\tilde{V}(s) & =\frac{e^{\alpha(s-t)}}{\sqrt{S_{\alpha, w, v}(s-t)}} v \\
\tilde{X}(s) & =x-\frac{1}{\alpha} \log \left(\frac{\|\tilde{V}(s)\| e^{\alpha(t-s)}+w \sqrt{S_{\alpha, w, \tilde{V}(s)}(t-s)}}{\|\tilde{V}(s)\|+w}\right) \frac{w}{\|\tilde{V}(s)\|} \tilde{V}(s),
\end{aligned}
$$

so that

$$
f(t, x, v)=e^{-d \alpha t} f^{0}(\tilde{X}(0), \tilde{V}(0))\left(1+\left(e^{2 \alpha t}-1\right) \frac{\|\tilde{V}(0)\|^{2}}{w^{2}}\right)^{1+d / 2} .
$$

We will always consider compactly supported initial data in $v$, meaning that we can put a homogeneous Dirichlet condition at the boundaries. Note that the stability condition we have to adopt is therefore

$$
2 \alpha \Delta t<\log \left(\beta\left\|v_{\max }\right\|^{2} /\left(\beta\left\|v_{\max }\right\|^{2}-1\right)\right) .
$$




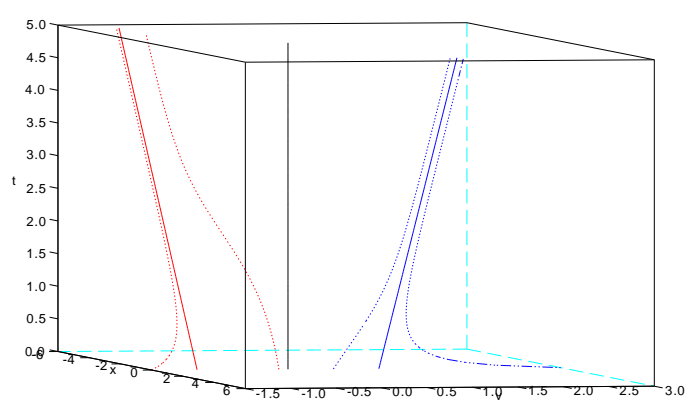

(a) $3 \mathrm{D}$ view

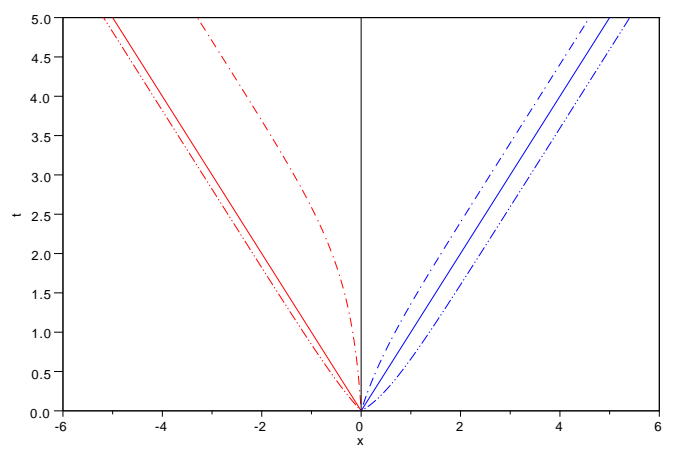

(c) $(v, t)$ projection

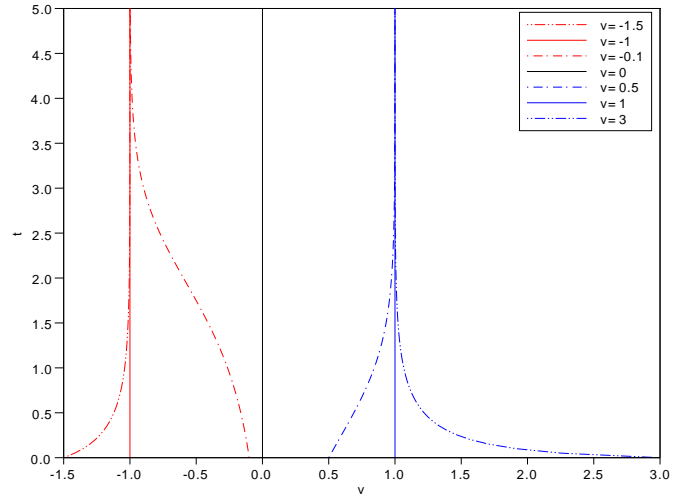

(b) $(x, t)$ projection

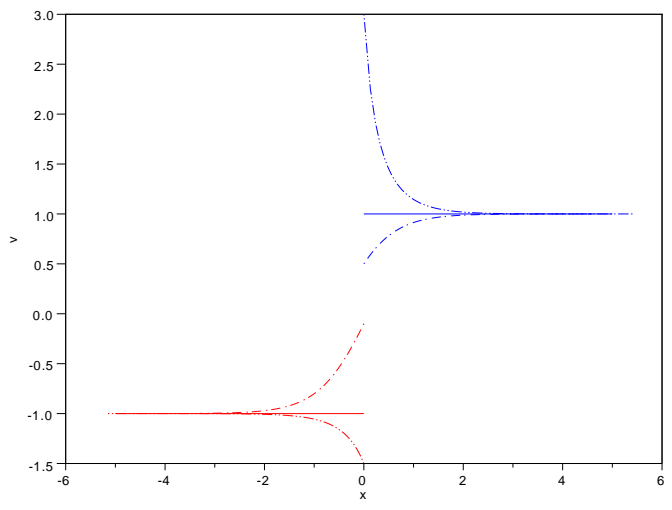

(d) Projection in the phase space

Figure 22: Plot of seven characteristic curves including only the advection terms in $x$ and $v$ in $1 \mathrm{D}\left(x^{0}=0\right.$ and $\left.v^{0} \in\{-1.5,-1,-0.1,0,0.5,1,3\}\right)$ 


\section{References}

[1] F. Cucker, S. Smale, D.-X. Zhou, Modeling language evolution, Found. Comput. Math. 4 (3) (2004) 315-343. doi:10.1007/s10208-003-0101-2.

URL http://dx.doi.org/10.1007/s10208-003-0101-2

[2] J. Toner, Y. Tu, Long-range order in a two-dimensional dynamical xy model: How birds fly together, Phys. Rev. Lett. 75 (1995) 4326-4329.

[3] F. Cucker, S. Smale, Emergent behavior in Hocks, IEEE Trans. Automat. Control 52 (5) (2007) 852-862. doi:10.1109/TAC.2007.895842.

URL http://dx.doi.org/10.1109/TAC.2007.895842

[4] J. Parrish, S. Viscido, D. Gruenbaum, Self-organized fish schools: An examination of emergent properties, Biol. Bull. 202 (2002) 296-305.

[5] H. Niwa, Self-organizing dynamic model of fish schooling, J. Theor. Biol. 171 (1994) $123-136$.

[6] W. Romey, Individual differences make a difference in the trajectories of simulated schools of fish, Ecol. Model. 92 (1996) 65-77.

[7] I. Couzin, N. Franks, Self-organized lane formation and optimized traffic flow in army ants, Proc. R. Soc. Lond. B 270 (2002) 139-146.

[8] A. Koch, D. White, The social lifestyle of myxobacteria, Bioessays 20 (1998) 1030-1038.

[9] Y. Chuang, Y. Huang, M. D’Orsogna, A. Bertozzi, Multi-vehicle flocking: scalability of cooperative control algorithms using pairwise potentials, IEEE International Conference on Robotics and Automation (2007) 2292-2299.

[10] L. Perea, G. Gómez, P. Elosegui, Extension of the Cucker-Smale control law to space flight formations, AIAA Journal of Guidance, Control, and Dynamics 32 (2009) 527-537.

[11] A. Jadbabaie, J. Lin, A. S. Morse, Correction to: "Coordination of groups of mobile autonomous agents using nearest neighbor rules" |IEEE Trans. Automat. Control 48 (2003), no. 6, 988-1001; MR 1986266 IEEE Trans. Automat. Control 48 (9) (2003) 1675. doi:10.1109/TAC.2003.817537.

URL http://dx.doi.org/10.1109/TAC.2003.817537

[12] E. F. N.E. Leonard, Virtual leaders, artificial potentials and coordinated control of groups, Proc. 40th IEEE Conf. Decision Contr. (2001) 2968-2973.

[13] M. S. K. Sugawara, Cooperative acceleration of task performance: Foraging behavior of interacting multi-robots system, Physica D 100 (1997) 343-354. 
[14] M. B. Short, M. R. D'Orsogna, V. B. Pasour, G. E. Tita, P. J. Brantingham, A. L. Bertozzi, L. B. Chayes, A statistical model of criminal behavior, Math. Models Methods Appl. Sci. 18 (suppl.) (2008) 1249-1267. doi:10.1142/S0218202508003029. URL http://dx.doi.org/10.1142/S0218202508003029

[15] A. Mogilner, L. Edelstein-Keshet, L. Bent, A. Spiros, Mutual interactions, potentials, and individual distance in a social aggregation, J. Math. Biol. 47 (4) (2003) 353-389. doi:10.1007/s00285-003-0209-7. URL http://dx.doi.org/10.1007/s00285-003-0209-7

[16] H. Levine, W. Rappel, I. Cohen, Self-organization in systems of self-propelled particles, Phys. Rev. E 63.

[17] M. R. D’Orsogna, Y. L. Chuang, A. L. Bertozzi, L. S. Chayes, Selt-propelled particles with soft-core interactions: Patterns, stability, and collapse, Physical Review Letters 96 (10) (2006) 104302. doi:10.1103/PhysRevLett.96.104302.

URL http://link.aps.org/abstract/PRL/v96/e104302

[18] G. Flierl, D. Gruenbaum, S. Levin, D. Olson, From individuals to aggregations: The interplay between behavior and physics, J. Theor. Biol. 196 (1999) 397-454.

[19] T. C. M., A. L. Bertozzi, M. A. Lewis, A nonlocal continuum model for biological aggregation, Bull. Math. Biol. 68 (7) (2006) 1601-1623. doi:10.1007/s11538-006-9088-6. URL http://dx.doi.org/10.1007/s11538-006-9088-6

[20] C. M. Topaz, A. L. Bertozzi, Swarming patterns in a two-dimensional kinematic model for biological groups, SIAM J. Appl. Math. 65 (1) (2004) 152-174 (electronic). doi: 10.1137/S0036139903437424.

URL http://dx.doi.org/10.1137/S0036139903437424

[21] E. Bertin, M. Droz, G. Grégoire, Boltzmann and hydrodynamic description for selfpropelled particles, Phys. Rev. E 74 .

[22] J. H. Irving, J. G. Kirkwood, The statistical mechanical theory of transport processes. IV. The equations of hydrodynamics, J. Chem. Phys. 18 (1950) 817-829.

[23] M. Burger, V. Capasso, D. Morale, On an aggregation model with long and short range interactions, Nonlinear Anal. Real World Appl. 8 (3) (2007) 939-958. doi:10.1016/j. nonrwa.2006.04.002.

URL http://dx.doi.org/10.1016/j.nonrwa.2006.04.002

[24] P. Degond, S. Motsch, Continuum limit of self-driven particles with orientation interaction, Math. Models Methods Appl. Sci. 18 (suppl.) (2008) 1193-1215. doi: 10.1142/S0218202508003005.

URL http://dx.doi.org/10.1142/S0218202508003005 
[25] P. Degond, S. Motsch, Large scale dynamics of the persistent turning walker model of tish behavion, J. Stat. Phys. 131 (6) (2008) 989-1021. doi:10.1007/ s109bb-008-9b29-8.

URL http://dx.doi.org/10.1007/s10955-008-9529-8

[26] S.-Y. Ha, E. Tadmor, From particle to kinetic and hydrodynamic descriptions of flocking, Kinet. Relat. Models 1 (3) (2008) 415-435.

[27] J. A. Carrillo, M. R. D’Orsogna, V. Panferov, Double milling in selt-propelled swarms from kinetic theory, Kinet. Relat. Models 2 (2) (2009) 363-378. doi:10.3934/krm. 2009.2 .363$.

URL http://dx.doi.org/10.3934/krm.2009.2.363

[28] J. A. Carrillo, M. Fornasier, J. Rosado, G. Toscani, Asymptotic Hocking dynamics for the kinetic Cucker-Smale model, SIAM J. Math. Anal. 42 (1) (2010) 218-236. doi: 10.1137/090757290.

URL http://dx.doi.org/10.1137/090757290

[29] M. Bostan, J. A. Carrillo, Asymptotic Fixed-Speed Reduced Dynamics for Kinetic Equations in Swarming, preprint (2012).

URL http://arxiv.org/abs/1202.6557

[30] T. Vicsek, A. Czirok, E. Ben-Jacob, I. Cohen, O. Shochet, Novel type of phase transition in a system of self-driven particles, Phys. Rev. Lett. 75 (1995) 1226-1229.

[31] S.-Y. Ha, J.-G. Liu, A simple proof of the Cucker-Smale Hocking dynamics and meanfield limit, Commun. Math. Sci. 7 (2) (2009) 297-325.

URL http://projecteuclid.org/getRecord?id=euclid.cms/1243443982

[32] J. A. Cañizo, J. A. Carrillo, J. Rosado, A well-posedness theory in measures for some kinetic models of collective motion, Math. Mod. Meth. Appl. Sci.

[33] M. Fornasier, J. Haskovec, G. Toscani, Fluid dynamic description of flocking via Povzner-Boltzmann equation, Physica D (nonlinear phenomena) 240 (1) (2011) 21-31.

[34] Y. Chuang, M. D’Orsogna, D. Marthaler, A. Bertozzi, L. Chayes, State transition and the continuum limit for the 2D interacting, self-propelled particle system, Physica D 232 (2007) 33-47.

[35] J. A. Carrillo, M. Fornasier, G. Toscani, F. Vecil, Particle, kinetic, and hydrodynamic models of swarming, in: G. Naldi, L. Pareschi, G. Toscani, N. Bellomo (Eds.), Mathematical Modeling of Collective Behavior in Socio-Economic and Life Sciences, Modeling and Simulation in Science, Engineering and Technology, Birkhäuser Boston, 2010, pp. $297-336$.

[36] J. A. Carrillo, M. R. D’Orsogna, V. Panferov, Double milling in self-propelled swarms from kinetic theory, Kinetic and Related Models 2 (2009) 363-378. 
[37] J. Rayleigh, The Theory of Sound, MacMillan London (2nd edition) 1.

[38] A. S. Mikhailov, D. Zanette, Noise-induced breakdown of coherent collective motion in swarms, Phys. Rev. E 60 (1999) 4571-4575.

[39] U. Erdmann, W. Ebeling, V. S. Anishchenko, Excitation of rotational modes in twodimensional systems of driven Brownian particles, Phys. Rev. E (3) 65 (6) (2002) 061106, 9. doi:10.1103/PhysRevE.65.061106.

URL http://dx.doi.org/10.1103/PhysRevE.65.061106

[40] W. Ebeling, U. Erdmann, Nonequilibrium statistical mechanics of swarms of driven particles, Complexity 8 (4) (2003) 23-30, complex adaptive systems, Part II. doi: $10.1002 / \mathrm{cplx} .10090$.

URL http://dx.doi.org/10.1002/cplx.10090

[41] I. Aoki, Simulation Study of the Schooling Mechanism in Fish., Bulletin of the Japanese Society of Scientific Fisheries 48 (1982) 1081-1088.

[42] A. Huth, C. Wissel, The simulation of the Movement of Fish Schools, Journal of Theoretical Biology.

[43] D. Ruelle, Statistical Mechanics, Rigorous Results, W.A. Benjamin Inc.

[44] F. Filbet, E. Sonnendrücker, P. Bertrand, Conservative numerical schemes for the Vlasov equation, J. Comput. Phys. 172 (1) (2001) 166-187. doi:10.1006/jcph.2001.6818. URL http://dx.doi.org/10.1006/jcph.2001.6818

[45] G. Strang, On the construction and comparison of difference schemes, SIAM J. Numer. Anal. 5 (1968) 506-517.

[46] C. Cheng, G. Knorr, The integration of the Vlasov equation in configuration space, J. Comput. Phys. 22 (1976) 330-351.

[47] J. Stoer, R. Bulirsch, Introduction to numerical analysis, 3rd Edition, Vol. 12 of Texts in Applied Mathematics, Springer-Verlag, New York, 2002, translated from the German by R. Bartels, W. Gautschi and C. Witzgall. 